Supporting information for:

\title{
9-Acylation of 1-Acyldipyrromethanes Containing a Dialkylboron Mask for the $\mathrm{C}$-Acylpyrrole Motif
}

H. Z. Syeda Huma, Kannan Muthukumaran, Shun-ichi Tamaru, and Jonathan S. Lindsey

\section{Table of Contents}

1. Refined synthesis of Mukaiyama reagents

S1-S1

2. Studies of the acylation of a dipyrromethane

S1-S4

3. Experimental Section

S5-S10

4. X-ray crystallographic analysis of $\mathbf{7 b}$

S11-S17

5. Spectral data for selected compounds

S18-S28

\section{Refined Synthesis of Mukaiyama Reagents.}

One procedure originally reported by Mukaiyama for the synthesis of $S$-2-pyridyl thioates entails reaction of equimolar amounts $(0.67 \mathrm{M}$ each) of an acid chloride, 2-mercaptopyridine, and TEA in THF for $4 \mathrm{~h}$. The TEA $\mathrm{HCl}$ was removed by filtration. Concentration of the filtrate afforded the crude $S$-2-pyridyl thioate product, which could be purified by distillation or recrystallization. ${ }^{6}$ We previously modified this method whereby the acid chloride and 2mercaptopyridine were reacted in $\mathrm{CH}_{2} \mathrm{Cl}_{2}(0.14 \mathrm{M}$ each). Addition of aqueous $\mathrm{NaOH}$, separation of the organic layer, and concentration of the organic layer afforded the product (often as an oil). Dissolution in ethyl acetate and addition of hexanes afforded the desired $S$-2-pyridyl thioate. ${ }^{3}$

We have now modified the method to facilitate large-scale preparation of the Mukaiyama reagents. The reaction of an acid chloride and 2-mercaptopyridine is carried out in THF (0.5-1.0 $\mathrm{M}$ each) at room temperature in the absence of any base. The desired $S$-2-pyridyl thioate precipitates as the $\mathrm{HCl}$ salt. Subsequent dissolution in diethyl ether/aqueous $\mathrm{NaHCO}_{3}$, concentration of the diethyl ether layer, and addition of hexanes afforded the desired $S$-2-pyridyl thioate as a precipitate. Application of this method to various substrates consistently gave yields of $\sim 90 \%$, affording the known Mukaiyama reagents $\mathbf{2 a}-\mathbf{d}^{1}$ and $\mathbf{2} \mathrm{e}^{1,3}$ in analytically pure form (Scheme 3). The procedure has been scaled linearly in one case to give the Mukaiyama reagent in substantial quantity ( $22 \mathrm{~g}, 96 \%$ yield).

\section{Studies of the acylation of a dipyrromethane.}

1-Acylation of a dipyrromethane. The success of the method developed for 9-acylation of a 1-acyldipyrromethane, which employs mesitylmagnesium bromide (MesMgBr), prompted us to investigate the 1-acylation of a dipyrromethane using $\mathrm{MesMgBr}$ rather than $\mathrm{EtMgBr}$ (Scheme S1). The reaction of 5-phenyldipyrromethane (1a) was first performed under the standard conditions for 1-acylation (entry 1, Table S1). Thus, a sample of 1a was treated with 2 molar equiv of $\mathrm{EtMgBr}$ in THF at room temperature for $10 \mathrm{~min}$. The solution was cooled to $\square 78$ ${ }^{\circ} \mathrm{C}$, whereupon 1 molar equiv of the Mukaiyama reagent 2a was added. After 10 min, the reaction mixture was quenched by addition of saturated aqueous $\mathrm{NH}_{4} \mathrm{Cl}$. Separation of the phases, concentration of the organic layer, and examination of the crude organic product by ${ }^{1} \mathrm{H}$ NMR spectroscopy revealed the extent of acylation. 
<smiles>c1ccc(C(c2ccc[nH]2)c2ccc[nH]2)cc1</smiles>

$1 a$
(1) RMgBr, THF $\mathrm{T}_{1}, 10 \mathrm{~min}$

(2) Acylating agent, THF $\mathrm{T}_{2}, 10 \mathrm{~min}$

$$
\mathrm{Ar}=p \text {-tolyl }
$$

Acylating agent:<smiles>Cc1ccc(C(=O)Sc2ccccn2)cc1</smiles>

2a<smiles>O=C([Al])c1ccc(C(c2ccccc2)c2ccc[nH]2)[nH]1</smiles>

$3 a$<smiles>Cc1ccc(C(=O)Cl)cc1</smiles>

$11 \mathrm{a}$

\section{Scheme S1}

The diagnostic feature for acylation is provided in the spectral region of 5-6 ppm, where each meso-proton $\left(\mathrm{H}^{5}\right)$ from the starting dipyrromethane, the target 1-acyldipyrromethane, and the undesired 1,9-diacyldipyrromethane is readily observable (Figure S1). The dipyrromethane (1a), uncomplexed 1-acyldipyrromethane (3a), or the 1,9-diacyldipyrromethane (4a) gives a peak for the meso-proton $\left(\mathrm{H}^{5}\right)$ at $5.49 \mathrm{ppm}, \square 5.57 \mathrm{ppm}$, or $\square 5.68 \mathrm{ppm}$ respectively. Integration of the peak areas gives a direct measure of the extent of acylation. (Note that the $\mathrm{H}^{5}$ proton provides a superb diagnostic for the uncomplexed dipyrromethane species, while in the studies using a dialkylboron complex, the peak stemming from the $\mathrm{H}^{5}$ proton of the 1 acyldipyrromethane- $\mathrm{BR}_{2}$ complex overlaps with that for the $\mathrm{H}^{7}$ proton of the 1,9diacyldipyrromethane- $\mathrm{BR}_{2}$ complex. With the studies using a dialkylboron complex, analysis of the crude reaction sample is best achieved by comparing the peak intensity for the $\mathrm{H}^{3}$ proton signals for the 1,9-diacylated $(\square 6.46 \mathrm{ppm})$ and 1-acylated $(\square 6.41 \mathrm{ppm})$ dipyrromethane-BR $\mathrm{B}_{2}$ complexes.

For the standard acylation method, the desired 1-acyldipyrromethane is present in $\sim 85 \%$ relative yield, with the remaining material consisting of unreacted dipyrromethane and no detectable 1,9-diacyldipyrromethane (entry 1, Table S1). Use of mesitylmagnesium bromide in place of EtMgBr gave no improvement in yield (entry 2). Use of $p$-toluoyl chloride with $\mathrm{EtMgBr}$ in the same molar quantities gave nearly equal amounts of unreacted starting material and 1-acyldipyrromethane (entry 3). Use of $p$-toluoyl chloride with $\mathrm{MesMgBr}$ in the same molar quantities gave a significant amount of the desired 1-acyldipyrromethane along with unreacted starting material and 1,9-diacyldipyrromethane (entry 4). Thus, this limited study gave no improvement over the standard conditions for 1-acylation. 
Figure S1. Expanded region of the ${ }^{1} \mathrm{H}$ NMR spectrum of the crude sample from the reaction of 1a $(1 \mathrm{mmol})$ with $\mathrm{Mes} \mathrm{MgBr}(2 \mathrm{mmol})$ in THF followed by treatment with a solution of 11a (1 $\mathrm{mmol}$ ) in THF at $\square 78^{\circ} \mathrm{C}$ (Table S1, entry 4).

Table S1. Study of the 1-acylation of dipyrromethane 1a. ${ }^{a}$

\begin{tabular}{|c|c|c|c|c|c|}
\hline Entry & Grignard Reagent $^{b}$ & $\mathrm{~T}_{1}$ & Acylating Agent $^{c}$ & $\mathrm{~T}_{2}$ & Ratio of 1a:3a:4a $^{d}$ \\
\hline 1 & EtMgBr & $\mathrm{rt}$ & $\mathbf{2 a}$ & $-78^{\circ} \mathrm{C}$ & $1: 7: 0$ \\
\hline 2 & MesMgBr & $\mathrm{rt}$ & $\mathbf{2 a}$ & $-78^{\circ} \mathrm{C}$ & $1: 4: 0$ \\
\hline 3 & EtMgBr & $\mathrm{rt}$ & $\mathbf{1 1 a}$ & $-78^{\circ} \mathrm{C}$ & $4: 5: 0$ \\
\hline 4 & $\mathrm{MesMgBr}$ & $\mathrm{rt}$ & $\mathbf{1 1 a}$ & $-78^{\circ} \mathrm{C}$ & $2.3: 10.1: 1$ \\
\hline
\end{tabular}

${ }^{a}$ Reactions were performed with $1 \mathrm{mmol}$ of $1 \mathrm{a}$ in THF $(1 \mathrm{~mL})$ under argon to which was added 2 mmol of Grignard reagent at room temperature $\left(T_{1}\right)$ for $10 \mathrm{~min}$, followed by cooling $\left(T_{2}\right)$ and treatment with $1 \mathrm{mmol}$ of acylating agent for $10 \mathrm{~min} .{ }^{b} \mathrm{~A} 1 \mathrm{M}$ solution of Grignard reagent in THF was used. ${ }^{c}$ The acylating agent was added as a $1 \mathrm{M}$ solution in THF. ${ }^{d}$ The ratio of 1a:3a:4a was determined from ${ }^{1} \mathrm{H}$ NMR spectra of crude samples (see text).

While no improvement in the yield of 1-acyldipyrromethane was achieved, it is worthwhile to consider why this reaction works at all. An explanation for the efficacy of the 1acylation of dipyrromethanes is as follows. Use of 2 equiv of EtMgBr per dipyrromethane in effect provides the 1 equiv of buffering capacity needed for the complete introduction of one acyl group (eq S1). Upon 1-acylation, the liberated HX is neutralized by the more basic pyrrole unit, which is the unacylated pyrrole half of the 1-acyldipyrromethane (eq S2). While the situation may be more complex in picture, involving aggregation phenomena, simple 
consideration of stoichiometry and acid-base properties of the reacting species would appear to explain the selective formation of the 1-acyldipyrromethane species.

(eq S1)

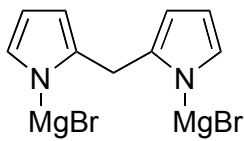

(eq S2)

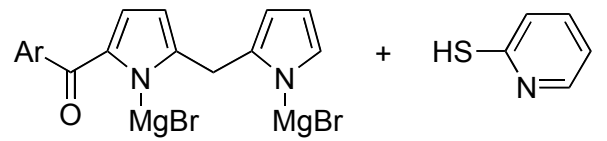

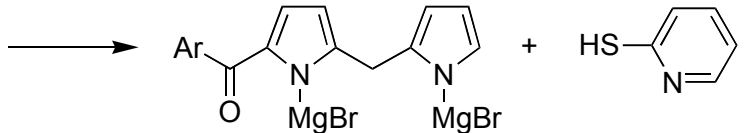

$\left.\longrightarrow \prod_{\substack{1 \\ M g B r}}\right|_{\substack{1 \\ H}} ^{\mid}$

1,9-Diacylation of a dipyrromethane. A series of experiments also was performed to determine whether direct 1,9-diacylation could be achieved in a superior fashion using $\mathrm{MesMgBr}$ rather than the standard conditions using EtMgBr. The standard conditions employ reaction at room temperature using the dipyrromethane 1a, ethylmagnesium bromide, and $p$-toluoyl chloride (11a) in a ratio of 1:5:2.5 in toluene. ${ }^{1}$ This experiment is listed as entry 1 (Table S2). For comparison, the same reaction in THF affords a lower yield of diacyldipyrromethane (entry 2). The reaction in toluene using the Mukaiyama reagent 2a instead of $p$-toluoyl chloride also gave even less diacylation (entry 3). Similar reaction of $\mathbf{1 a}$ using MesMgBr and 2a in THF gave a mixture consisting of significant amounts of 1-acyldipyrromethane and 1,9diacyldipyrromethane as well as a trace of unreacted dipyrromethane (entry 4). The final two reactions employed stoichiometric quantities of Grignard reagent (4 molar equiv) and acylating agent ( 2 molar equiv) relative to the dipyrromethane. Thus, reaction of 1 a with $\mathrm{MesMgBr}$ and the Mukaiyama reagent 2a gave equal amounts of the 1-acyldipyrromethane and the 1,9diacyldipyrromethane (entry 5). On the other hand, reaction of 1a with MesMgBr and $p$-toluoyl chloride (11a) gave a significant increase in the amount of 1,9-diacyldipyrromethane relative to the 1-acyldipyrromethane (entry 6). Although the relative yield of 1,9-diacyldipyrromethane was reasonable in entry 6 , the best conditions identified remain those of entry 1 , the standard conditions for 1,9-diacylation.

Table S2. Study of the 1,9-diacylation of dipyrromethane 1a. ${ }^{\text {a }}$

\begin{tabular}{|c|c|c|c|c|c|}
\hline Entry & $\begin{array}{l}\text { Grignard } \\
\text { reagent }^{b}\end{array}$ & $\begin{array}{l}\text { Acylating } \\
\text { agent }^{c}\end{array}$ & $\begin{array}{c}\text { Reactant ratio } \\
\text { DPM:RMgBr:RCOX }\end{array}$ & $\begin{array}{c}\text { Reaction } \\
\text { solvent }\end{array}$ & Ratio of 1a:3a:4a \\
\hline 1 & EtMgBr & $11 \mathrm{a}$ & $1: 5: 2.5$ & toluene & $0: 1: 5$ \\
\hline 2 & EtMgBr & $11 \mathrm{a}$ & $1: 5: 2.5$ & THF & $0: 1: 1.9$ \\
\hline 3 & EtMgBr & $2 a$ & $1: 5: 2.2$ & toluene & $0: 2.1: 1.0$ \\
\hline 4 & MesMgBr & $2 \mathbf{a}$ & $1: 2.5: 2.2$ & THF & $1: 30: 10$ \\
\hline 5 & MesMgBr & $\mathbf{2 a}$ & $1: 4: 2$ & THF & $0: 1: 1.1$ \\
\hline 6 & MesMgBr & $11 a$ & $1: 4: 2$ & THF & $0: 1: 3.5$ \\
\hline
\end{tabular}

${ }^{a}$ Reactions were performed with $1 \mathrm{mmol}$ of $1 \mathrm{a}$ in the specified solvent $(1 \mathrm{~mL})$ under argon to which was added the Grignard reagent at room temperature for $10 \mathrm{~min}$, followed by treatment with the acylating agent for $10 \mathrm{~min}$ at room temperaure. ${ }^{b} \mathrm{~A} 1 \mathrm{M}$ solution of Grignard reagent in THF was used. ${ }^{c}$ The acylating agent was added as a $1 \mathrm{M}$ solution in THF. ${ }^{d}$ The ratio of 1a:3a:4a was determined from ${ }^{1} \mathrm{H}$ NMR spectra of crude samples (see text). 


\section{Experimental Section}

General. ${ }^{1} \mathrm{H}$ NMR $(400 \mathrm{MHz})$ and ${ }^{13} \mathrm{C}$ NMR $(100 \mathrm{MHz})$ spectra were collected routinely in $\mathrm{CDCl}_{3}$ unless noted otherwise. Melting points are uncorrected. Silica gel $(40 \square \mathrm{m}$ average particle size) was used for column chromatography. THF was distilled from sodium under argon. Toluene and $\mathrm{CH}_{2} \mathrm{Cl}_{2}$ (reagent grade) were used as received. All other chemicals were reagent grade and were used as received. The 1-acyldipyrromethanes, 1,9dipyrromethanes, 1-acyldipyrromethane- $\mathrm{BR}_{2}$ complexes and 1,9-diacylpyrromethane- $\mathrm{BR}_{2}$ complexes are easily detected in TLC upon exposure to $\mathrm{Br}_{2}$ vapor.

S-2-Pyridyl 4-methoxybenzothioate (2b). ${ }^{1}$ Following the general procedure described for 2a, reaction of $p$-anisoyl chloride $(4.70 \mathrm{~g}, 30.0 \mathrm{mmol}$ ) with 2-mercaptopyridine (3.33 g, 30.0 $\mathrm{mmol})$ in THF $(30 \mathrm{~mL})$ for $30 \mathrm{~min}$ at room temperature afforded a pale yellow solid after precipitation $(6.70 \mathrm{~g}, 91 \%): \mathrm{mp} 73{ }^{\circ} \mathrm{C} ;{ }^{1} \mathrm{H}$ NMR $\mathrm{C} 3.88(\mathrm{~s}, 3 \mathrm{H}), 6.93-7.00(\mathrm{~m}, 2 \mathrm{H}), 7.30-7.34$ (m, 1H), 7.70-7.74 (m, 2H), 7.75-7.80 (m, 1H), 7.97-8.02 (m, 1H), 8.64-8.69 (m, 1H); Anal. Calcd for $\mathrm{C}_{13} \mathrm{H}_{11} \mathrm{NO}_{2} \mathrm{~S}: \mathrm{C}, 63.65 ; \mathrm{H}, 4.52 ; \mathrm{N}, 5.71$. Found: $\mathrm{C}, 63.78 ; \mathrm{H}, 4.59 ; \mathrm{N}, 5.66$.

$S$-2-Pyridyl pentafluorobenzothioate (2c). ${ }^{1}$ Following the general procedure described for $2 \mathrm{a}$, reaction of pentafluorobenzoyl chloride $(6.92 \mathrm{~g}, 30.0 \mathrm{mmol})$ with 2-mercaptopyridine $(3.33 \mathrm{~g}, 30.0 \mathrm{mmol})$ in THF $(30 \mathrm{~mL})$ for $30 \mathrm{~min}$ at room temperature afforded a pale yellow solid after precipitation $(7.97 \mathrm{~g}, 87 \%)$ : $\mathrm{mp} 51{ }^{\circ} \mathrm{C} ;{ }^{1} \mathrm{H}$ NMR $\square 7.37-7.42(\mathrm{~m}, 1 \mathrm{H}), 7.75-7.79(\mathrm{~m}, 1 \mathrm{H})$, 7.81-7.87 (m, 1H), 8.67-8.71 (m, 1H); Anal. Calcd for $\mathrm{C}_{12} \mathrm{H}_{4} \mathrm{NOS}: \mathrm{C}, 47.22 ; \mathrm{H}, 1.32 ; \mathrm{N}, 4.59$. Found: C, 47.59; H, 1.29; N, 4.38.

S-2-Pyridyl 4-iodobenzothioate (2d). ${ }^{1}$ Following the general procedure described for 2a, reaction of 4-iodobenzoyl chloride $(8.01 \mathrm{~g}, 30.0 \mathrm{mmol})$ with 2-mercaptopyridine $(3.33 \mathrm{~g}$, $30.0 \mathrm{mmol})$ in THF $(60 \mathrm{~mL})$ for $30 \mathrm{~min}$ at room temperature afforded a pale yellow solid after precipitation (9.61 g, 94\%): $\mathrm{mp} 127{ }^{\circ} \mathrm{C} ;{ }^{1} \mathrm{H}$ NMR $\square 7.30-7.38(\mathrm{~m}, 1 \mathrm{H}), 7.70-7.76(\mathrm{~m}, 3 \mathrm{H})$, 7.77-7.83 (m, 1H), 7.84-7.89 (m, 2H), 8.64-8.71 (m, 1H); Anal. Calcd for $\mathrm{C}_{12} \mathrm{H}_{8} \mathrm{INOS}: \mathrm{C}$, 42.25; H, 2.36; N, 4.11. Found: C, 42.27; H, 2.38; N, 4.05 .

$\boldsymbol{S}$-2-Pyridyl 4-bromobenzothioate (2e) ${ }^{1,3}$ Following the general procedure described for 2a, reaction of 4-bromobenzoyl chloride $(13.2 \mathrm{~g}, 60.0 \mathrm{mmol})$ with 2-mercaptopyridine (6.67 $\mathrm{g}, 60.0 \mathrm{mmol})$ in THF $(120 \mathrm{~mL})$ afforded a pale yellow solid $(16.2 \mathrm{~g}, 92 \%): \mathrm{mp} 134{ }^{\circ} \mathrm{C} ;{ }^{1} \mathrm{H}$ NMR $\square 7.30-7.39(\mathrm{~m}, 1 \mathrm{H}), 7.62-7.68(\mathrm{~m}, 2 \mathrm{H}), 7.70-7.78(\mathrm{~m}, 1 \mathrm{H}), 7.79-7.84(\mathrm{~m}, 1 \mathrm{H}), 7.86-7.92$ $(\mathrm{m}, 2 \mathrm{H}), 8.64-8.72(\mathrm{~m}, 1 \mathrm{H})$; Anal. Calcd for $\mathrm{C}_{12} \mathrm{H}_{8} \mathrm{BrNOS}: \mathrm{C}, 49.00 ; \mathrm{H}, 2.74 ; \mathrm{N}, 4.76$. Found: C, 49.28; H, 2.70; N, 4.83 .

\section{0-(9-Borabicyclo[3.3.1]non-9-yl)-9-(p-methoxybenzoyl)-5-phenyl-1-p-}

toluoyldipyrromethane (7b). Following the general procedure (method A) described for $7 \mathbf{a}$, reaction of $6 \mathbf{a}(0.92 \mathrm{~g}, 2.0 \mathrm{mmol})$ in THF $(2.2 \mathrm{~mL})$ with $\mathrm{MesMgBr}(4.0 \mathrm{~mL}, 4.0 \mathrm{mmol}, 1 \mathrm{M}$ solution in THF) followed by $\mathbf{2 b}(0.54 \mathrm{~g}, 2.2 \mathrm{mmol})$ in THF $(2.2 \mathrm{~mL})$ afforded a yellow powder $\left.(0.90 \mathrm{~g}, 76 \%): \mathrm{mp} 192{ }^{\circ} \mathrm{C}(\mathrm{dec})\right)^{1} \mathrm{H}$ NMR $\square 0.68 \square 0.74(\mathrm{~m}, 2 \mathrm{H}), 1.66 \square 2.26(\mathrm{~m}, 12 \mathrm{H}), 2.48(\mathrm{~s}$, $3 \mathrm{H}), 3.87(\mathrm{~s}, 3 \mathrm{H}), 6.00 \square 6.02(\mathrm{~m}, 1 \mathrm{H}), 6.10(\mathrm{~s}, 1 \mathrm{H}), 6.45(\mathrm{~d}, J=4.4 \mathrm{~Hz}, 1 \mathrm{H}), 6.80 \square 6.82(\mathrm{~m}, 1 \mathrm{H})$, $6.96(\mathrm{~d}, J=8.8 \mathrm{~Hz}, 2 \mathrm{H}), 7.19(\mathrm{~d}, J=8.8 \mathrm{~Hz}, 2 \mathrm{H}), 7.25 \square 7.39(\mathrm{~m}, 6 \mathrm{H}), 7.89(\mathrm{~d}, J=8.8 \mathrm{~Hz}, 2 \mathrm{H})$, $8.13(\mathrm{~d}, J=8.8 \mathrm{~Hz}, 2 \mathrm{H}), 9.12-9.20(\mathrm{br}, 1 \mathrm{H}) ;{ }^{13} \mathrm{C}$ NMR $\mathrm{Q} 22.1,23.8,25.1,26.2,30.7,31.0,34.5$, $34.6,45.0,55.6,111.2,113.7,118.4,119.5,120.8,127.5,128.1,128.4,128.9,129.9,130.0$, 131.08, 131.10, 131.3, 135.2, 140.1, 141.1, 145.3, 150.3, 162.8, 175.0, 183.4; FABMS obsd 595.3170, calcd 595.3132 [(M+H $\left.)^{+}\right],\left(\mathrm{M}=\mathrm{C}_{39} \mathrm{H}_{39} \mathrm{BN}_{2} \mathrm{O}_{3}\right)$; Anal. calcd for $\mathrm{C}_{39} \mathrm{H}_{39} \mathrm{BN}_{2} \mathrm{O}_{3}: \mathrm{C}$, 78.79; H, 6.61; N, 4.71. Found: C, 78.99; H, 6.75; N, 4.70. 


\section{0-(9-Borabicyclo[3.3.1]non-9-yl)-9-(pentafluorobenzoyl)-5-phenyl-1-p-}

toluoyldipyrromethane (7c). Following the general procedure (method A) described for 7a, reaction of $6 \mathbf{a}(0.92 \mathrm{~g}, 2.0 \mathrm{mmol})$ in $\mathrm{THF}(2 \mathrm{~mL})$ with $\mathrm{MesMgBr}(4.0 \mathrm{~mL}, 4.0 \mathrm{mmol}, 1 \mathrm{M}$ solution in THF) followed by $2 \mathrm{c}(0.67 \mathrm{~g}, 2.2 \mathrm{mmol})$ in THF $(2.2 \mathrm{~mL})$ afforded a yellow powder (1.07 g, 82\%): mp $195{ }^{\circ} \mathrm{C}$ (dec.); ${ }^{1} \mathrm{H}$ NMR $\square 0.66 \square 0.72(\mathrm{~m}, 2 \mathrm{H}), 1.66 \square 2.27(\mathrm{~m}, 12 \mathrm{H}), 2.49$ (s, $3 \mathrm{H}), 6.03 \square 6.05(\mathrm{~m}, 1 \mathrm{H}), 6.10(\mathrm{~s}, 1 \mathrm{H}), 6.44(\mathrm{~d}, J=4.4 \mathrm{~Hz}, 1 \mathrm{H}), 6.63 \square 6.64(\mathrm{~m}, 1 \mathrm{H}), 7.18(\mathrm{~d}, J=$ $8.0 \mathrm{~Hz}, 2 \mathrm{H}), 7.26 \square 7.39(\mathrm{~m}, 8 \mathrm{H}), 8.14(\mathrm{~d}, J=8.0 \mathrm{~Hz}, 2 \mathrm{H}), 9.03 \square 9.07$ (br, $1 \mathrm{H}) ;{ }^{13} \mathrm{C}$ NMR $\square 22.2$, 23.8, 25.1, 26.2, 26.4, 30.8, 30.9, 34.5, 34.6, 45.2, 112.4, 118.5, 120.6, 122.3, 127.9, 128.0, $128.5,129.3,130.0,130.1,131.2,135.4,140.2,144.1,145.7,148.9,171.9,175.7$; FABMS obsd 655.2600, calcd 655.2555 [(M+H) $\left.{ }^{+}\right],\left(\mathrm{M}=\mathrm{C}_{38} \mathrm{H}_{32} \mathrm{BF}_{5} \mathrm{~N}_{2} \mathrm{O}_{2}\right)$; Anal. calcd for $\mathrm{C}_{38} \mathrm{H}_{32} \mathrm{BF}_{5} \mathrm{~N}_{2} \mathrm{O}_{2}$ : $\mathrm{C}$, 69.74; H, 4.93; N, 4.28. Found: C, 69.97; H, 5.29; N, 4.21.

10-(9-Borabicyclo[3.3.1]non-9-yl)-1,9-di-p-toluoyldipyrromethane (7d). Following the general procedure (method A) described for $7 \mathbf{a}$, reaction of $\mathbf{6 b}(0.77 \mathrm{~g}, 2.0 \mathrm{mmol})$ in THF (2 $\mathrm{mL})$ with $\mathrm{MesMgBr}(4.0 \mathrm{~mL}, 4.0 \mathrm{mmol}, 1 \mathrm{M}$ solution in THF) followed by $2 \mathrm{a}(0.50 \mathrm{~g}, 2.20$ $\mathrm{mmol})$ in THF $(2.2 \mathrm{~mL})$ afforded a yellow suspension. The reaction mixture was filtered directly on a Buchner funnel. The filtrate (\#1) was set aside. The filtered material was stirred in a halfsaturated aqueous solution of $\mathrm{NH}_{4} \mathrm{Cl}(10 \mathrm{~mL})$ for $5 \mathrm{~min}$, affording a suspension. The suspension was filtered on a Buchner funnel. The filtered material was washed with $\mathrm{NaHCO}_{3}(20 \mathrm{~mL})$, water $(10 \mathrm{~mL})$ and $\mathrm{Et}_{2} \mathrm{O}(5 \mathrm{~mL})$ to afford a yellow powder $(0.80 \mathrm{mg}, 80 \%)$. Filtrate \#1 was worked up, affording additional material $(0.10 \mathrm{~g}, 10 \%)$. The combined yield $(0.90 \mathrm{~g})$ is $90 \%$ : mp $215{ }^{\circ} \mathrm{C}$ (dec.); ${ }^{1} \mathrm{H}$ NMR $\square 0.72 \square 0.79(\mathrm{~m}, 2 \mathrm{H}), 1.70 \square 2.28(\mathrm{~m}, 12 \mathrm{H}), 2.42$ (s, 3H), 2.48 (s, 3H), $4.45(\mathrm{~s}, 2 \mathrm{H}), 6.09 \square 6.10(\mathrm{~m}, 1 \mathrm{H}), 6.42(\mathrm{~d}, J=4.0 \mathrm{~Hz}, 1 \mathrm{H}), 6.82 \square 6.84(\mathrm{~m}, 1 \mathrm{H}), 7.25(\mathrm{~d}, J=8.0$ $\mathrm{Hz}, 2 \mathrm{H}), 7.31(\mathrm{~d}, J=4.0 \mathrm{~Hz}, 1 \mathrm{H}), 7.37(\mathrm{~d}, J=8.0 \mathrm{~Hz}, 2 \mathrm{H}), 7.78(\mathrm{~d}, J=8.0 \mathrm{~Hz}, 2 \mathrm{H}), 8.12(\mathrm{~d}, J=$

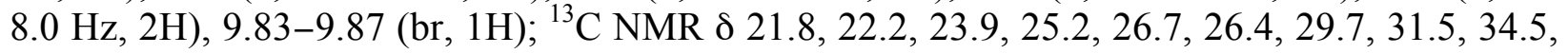
$110.5,118.5,120.5,120.7,128.2$, 129.2, 129.3, 129.9, 130.0, 130.9, 135.5, 135.8, 137.6, 142.5, 145.3, 147.2, 174.7, 184.3; FABMS obsd 503.2874, calcd 503.2870[(M+H $\left.)^{+}\right], \quad(\mathrm{M}=$ $\mathrm{C}_{33} \mathrm{H}_{35} \mathrm{BN}_{2} \mathrm{O}_{2}$ ). The elemental analysis for the sample assuming 0.5 molar equiv of water $\left(\mathrm{C}_{33} \mathrm{H}_{36} \mathrm{BN}_{2} \mathrm{O}_{2.5} ; \mathrm{C}, 77.49 ; \mathrm{H}, 7.09 ; \mathrm{N}, 5.48\right.$. Found: $\left.\mathrm{C}, 77.22 ; \mathrm{H}, 6.92 ; \mathrm{N}, 5.40\right)$ is in good agreement with the observed data.

10-(9-Borabicyclo[3.3.1]non-9-yl)-9-hexanoyl-1-p-toluoyldipyrromethane (7e). Following the general procedure (method A) described for 7a, reaction of $\mathbf{6 b}(0.77 \mathrm{~g}, 2.0 \mathrm{mmol})$ in THF $(2 \mathrm{~mL})$ with $\mathrm{MesMgBr}(4.0 \mathrm{~mL}, 4.0 \mathrm{mmol}, 1 \mathrm{M}$ solution in THF) followed by $2 \mathbf{f}(0.46 \mathrm{~g}$, $2.2 \mathrm{mmol})$ in THF $(2.2 \mathrm{~mL})$ afforded a yellow suspension. The reaction mixture was filtered directly on a Buchner funnel. The filtrate (\#1) was set aside. The filtered material was stirred in a half-saturated aqueous solution of $\mathrm{NH}_{4} \mathrm{Cl}(10 \mathrm{~mL})$ for $5 \mathrm{~min}$. The suspension was filtered on a Buchner funnel. The filtered material was washed with $\mathrm{NaHCO}_{3}(20 \mathrm{~mL})$, water $(10 \mathrm{~mL})$ and $\mathrm{Et}_{2} \mathrm{O}(5 \mathrm{~mL})$ to afford a yellow powder $(0.70 \mathrm{~g}, 73 \%)$. Filtrate \#1 after workup afforded additional material $(0.07 \mathrm{~g}, 7 \%)$. The combined yield $(0.77 \mathrm{~g})$ is $80 \%$ : $\mathrm{mp} 190{ }^{\circ} \mathrm{C}(\mathrm{dec}.) ;{ }^{1} \mathrm{H}$ NMR $\square 0.72 \square 0.76(\mathrm{~m}, 2 \mathrm{H}), 0.86 \square 0.90(\mathrm{~m}, 3 \mathrm{H}), 1.30 \square 1.36(\mathrm{~m}, 4 \mathrm{H}), 1.65 \square 2.27(\mathrm{~m}, 14 \mathrm{H}), 2.48(\mathrm{~s}$, $3 \mathrm{H}), 2.70(\mathrm{t}, J=8.0 \mathrm{~Hz}, 2 \mathrm{H}), 4.38(\mathrm{~s}, 2 \mathrm{H}), 6.02 \square 6.04(\mathrm{~m}, 1 \mathrm{H}), 6.36(\mathrm{~d}, J=4.0 \mathrm{~Hz}, 1 \mathrm{H})$, $6.84 \square 6.85(\mathrm{~m}, 1 \mathrm{H}), 7.30(\mathrm{~d}, J=4.4 \mathrm{~Hz}, 1 \mathrm{H}), 7.37(\mathrm{~d}, J=8.0 \mathrm{~Hz}, 2 \mathrm{H}), 8.11(\mathrm{~d}, J=8.0 \mathrm{~Hz}, 2 \mathrm{H})$,

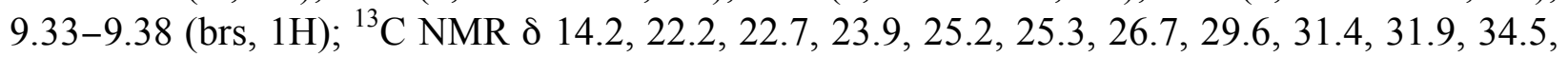
37.9, 109.9, 117.4, 118.4, 120.6, 128.2, 129.9, 130.2, 131.8, 135.5, 137.0, 145.2, 147.3, 174.6, 191.0; FABMS obsd 481.3199, calcd 483.3183 [(M+H $\left.{ }^{+}\right],\left(\mathrm{M}=\mathrm{C}_{31} \mathrm{H}_{39} \mathrm{BN}_{2} \mathrm{O}_{2}\right)$. Anal. calcd for $\mathrm{C}_{31} \mathrm{H}_{39} \mathrm{BN}_{2} \mathrm{O}_{2}$ : C, 77.17; H, 8.15; N, 5.81. Found: C, 76.94; H, 8.13; N, 5.73. 


\section{0-(9-Borabicyclo[3.3.1] non-9-yl)-9-( $p$-bromobenzoyl)-1-( $p$-methoxybenzoyl)-5-(p-}

methoxyphenyl)dipyrromethane (7f). Following the general procedure (method A) described for 7a, reaction of $\mathbf{6 c}(1.01 \mathrm{~g}, 2.0 \mathrm{mmol})$ in THF $(8 \mathrm{~mL}$, owing to solubility limitations even after adding the Grignard reagent) with $\mathrm{MesMgBr}(4.0 \mathrm{~mL}, 4.0 \mathrm{mmol}, 1 \mathrm{M}$ solution in THF) followed by $2 \mathrm{e}(0.65 \mathrm{~g}, 2.2 \mathrm{mmol})$ in THF $(8.8 \mathrm{~mL})$ afforded a yellow powder $(1.05 \mathrm{~g}, 76 \%)$ : $\mathrm{mp}$

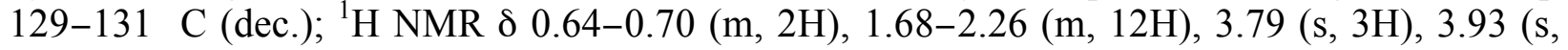
$3 \mathrm{H}), 5.99 \square 6.01(\mathrm{~m}, 1 \mathrm{H}), 6.03(\mathrm{~s}, 1 \mathrm{H}), 6.43(\mathrm{~d}, J=4.4 \mathrm{~Hz}, 1 \mathrm{H}), 6.76 \square 6.77(\mathrm{~m}, 1 \mathrm{H}), 6.85(\mathrm{~d}, J=$ $8.0 \mathrm{~Hz}, 2 \mathrm{H}), 7.06(\mathrm{~d}, J=8.0 \mathrm{~Hz}, 2 \mathrm{H}), 7.09(\mathrm{~d}, J=8.0 \mathrm{~Hz}, 2 \mathrm{H}), 7.31(\mathrm{~d}, J=4.0 \mathrm{~Hz}, 1 \mathrm{H}), 7.59$ (d, $J=8.0 \mathrm{~Hz}, 2 \mathrm{H}), 7.72(\mathrm{~d}, J=8.0 \mathrm{~Hz}, 2 \mathrm{H}), 8.22(\mathrm{~d}, J=8.0 \mathrm{~Hz}, 2 \mathrm{H}), 9.11 \square 9.16(\mathrm{brs}, 1 \mathrm{H}) ;{ }^{13} \mathrm{C}$ NMR $\square 23.9,25.2,26.2,30.8,31.0,34.6,34.7,44.3,55.5,55.9,114.4,114.8,117.9,120.3$, $120.5,123.4,129.5,130.7,131.7,132.2,133.1,134.8,137.3,142.0,149.6,158.9,164.7,174.5$, 183.2; FABMS obsd 689.2170, calcd 689.2188 [(M+H) $\left.{ }^{+}\right],\left(\mathrm{M}=\mathrm{C}_{39} \mathrm{H}_{38} \mathrm{BBrN}_{2} \mathrm{O}_{4}\right)$. Anal. calcd for $\mathrm{C}_{39} \mathrm{H}_{38} \mathrm{BBrN}_{2} \mathrm{O}_{4}$ : C, 67.94; $\mathrm{H}, 5.56 ; \mathrm{N}, 4.06$. Found: $\mathrm{C}, 68.05 ; \mathrm{H}, 5.66 ; \mathrm{N}, 4.09$.

\section{0-(9-Borabicyclo[3.3.1]non-9-yl)-1-p-hexanoyl-5-pentyl-9-p-toluoyldipyrromethane}

(7g). Following the general procedure (method A) described for 7a, reaction of $6 \mathbf{d}(0.87 \mathrm{~g}, 2.0$ $\mathrm{mmol})$ in THF (2 mL) with MesMgBr $(4.00 \mathrm{~mL}, 4.00 \mathrm{mmol}, 1 \mathrm{M}$ solution in THF) followed by 2a $(0.50 \mathrm{~g}, 2.2 \mathrm{mmol})$ in THF $(2 \mathrm{~mL})$ afforded an orange oil. Chromatography (silica, $\left.\mathrm{CH}_{2} \mathrm{Cl}_{2}\right)$ afforded an off-white solid $(0.78 \mathrm{~g}, 71 \%)$ : $\mathrm{mp} 130{ }^{\circ} \mathrm{C}$ (dec.); ${ }^{1} \mathrm{H}$ NMR $\square 0.54 \square 0.63$ (m, 2H), $0.82 \square 0.86(\mathrm{~m}, 3 \mathrm{H}), 0.92(\mathrm{t}, J=7.6 \mathrm{~Hz}, 3 \mathrm{H}), 1.24 \square 1.27(\mathrm{~m}, 6 \mathrm{H}), 1.34 \square 1.46(\mathrm{~m}, 6 \mathrm{H}), 1.64 \square 2.17$ $(\mathrm{m}, 14 \mathrm{H}), 2.42(\mathrm{~s}, 3 \mathrm{H}), 2.84(\mathrm{t}, J=7.6 \mathrm{~Hz}, 2 \mathrm{H}), 4.56(\mathrm{t}, J=7.6 \mathrm{~Hz}, 1 \mathrm{H}), 6.08 \square 6.11(\mathrm{~m}, 1 \mathrm{H}), 6.52$ $(\mathrm{d}, J=4.0 \mathrm{~Hz}, 1 \mathrm{H}), 6.77 \square 6.80(\mathrm{~m}, 1 \mathrm{H}), 7.09(\mathrm{~d}, J=4.0 \mathrm{~Hz}, 1 \mathrm{H}), 7.26(\mathrm{~d}, J=8.4 \mathrm{~Hz}, 2 \mathrm{H}), 7.76$ $(\mathrm{d}, J=8.4 \mathrm{~Hz}, 2 \mathrm{H}), 9.38-9.50(\mathrm{br}, 1 \mathrm{H}) ;{ }^{13} \mathrm{C} \mathrm{NMR} \square 14.1,14.2,21.7,22.5,22.6,23.9,25.0,25.5$, 25.9, 26.2, 27.5, 30.6, 31.1, 31.5, 31.8, 32.0, 34.1, 34.3, 36.7, 39.7, 109.5, 117.5, 117.6, 120.4, 129.1, 129.3, 130.8, 136.0, 136.7, 142.1, 142.3, 152.4, 184.4, 185.0; FABMS obsd 553.3963, calcd $553.3965\left[(\mathrm{M}+\mathrm{H})^{+}\right],\left(\mathrm{M}=\mathrm{C}_{36} \mathrm{H}_{49} \mathrm{BN}_{2} \mathrm{O}_{2}\right)$. Anal. calcd for $\mathrm{C}_{36} \mathrm{H}_{49} \mathrm{BN}_{2} \mathrm{O}_{2}: \mathrm{C}, 78.25 ; \mathrm{H}$, 8.94; N, 5.07. Found: C, 78.18; H, 9.00; N, 5.07.

\section{0-(9-Borabicyclo[3.3.1]non-9-yl)-1-(p-bromobenzoyl)-9-(p-iodobenzoyl)-5-[p-}

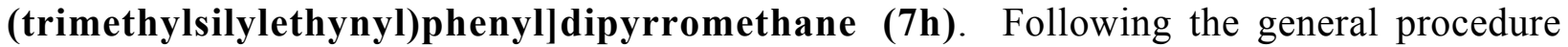
$(\operatorname{method} \mathrm{A})$ described for $7 \mathbf{a}$, reaction of $\mathbf{6 e}(1.2 \mathrm{~g}, 2.0 \mathrm{mmol})$ in THF $(2 \mathrm{~mL})$ with $\mathrm{MesMgBr}$ (4.00 mL, $4.00 \mathrm{mmol}, 1 \mathrm{M}$ solution in THF) followed by $2 \mathrm{~d}(0.75 \mathrm{~g}, 2.2 \mathrm{mmol})$ in THF (4 mL) afforded an orange oil. Chromatography [silica, $\mathrm{CH}_{2} \mathrm{Cl}_{2} /$ hexanes $(1: 1) \square \mathrm{CH}_{2} \mathrm{Cl}_{2} /$ hexanes $(2: 1)$ afforded a yellow-orange solid (1.26 g, 74\%): $\mathrm{mp} 139{ }^{\circ} \mathrm{C}$ (dec.); ${ }^{1} \mathrm{H}$ NMR $\square 0.25$ (s, 9H),

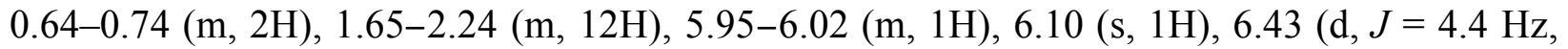
$1 \mathrm{H}), 6.75 \square 6.78(\mathrm{~m}, 1 \mathrm{H}), 7.04(\mathrm{~d}, J=8.0 \mathrm{~Hz}, 2 \mathrm{H}), 7.31(\mathrm{~d}, J=4.4 \mathrm{~Hz}, 1 \mathrm{H}), 7.36(\mathrm{~d}, J=8.0 \mathrm{~Hz}$, $2 \mathrm{H}), 7.51(\mathrm{~d}, J=8.0 \mathrm{~Hz}, 2 \mathrm{H}), 7.72(\mathrm{~d}, J=8.0 \mathrm{~Hz}, 2 \mathrm{H}), 7.80(\mathrm{~d}, J=8.0 \mathrm{~Hz}, 2 \mathrm{H}), 8.06(\mathrm{~d}, J=8.0$ $\mathrm{Hz}, 2 \mathrm{H}), 9.76-9.84$ (br, 1H); ${ }^{13} \mathrm{C}$ NMR $\square 0.18,23.6,25.0,26.2,30.6,31.0,34.4,34.6,44.9$, 95.0, $99.4,104.8,111.8,118.7,120.6,121.5,122.3,128.1,129.4,129.5,130.8,131.0,131.1,132.4$, 132.7, 135.2, 137.5, 137.6, 140.6, 141.4, 150.8, 173.6, 183.6; FABMS obsd 851.1362, calcd $851.1337\left[(\mathrm{M}+\mathrm{H})^{+}\right],\left(\mathrm{M}=\mathrm{C}_{42} \mathrm{H}_{41} \mathrm{BBrN}_{2} \mathrm{O}_{2} \mathrm{Si}\right)$. Anal. calcd for $\mathrm{C}_{42} \mathrm{H}_{41} \mathrm{BBrN}_{2} \mathrm{O}_{2} \mathrm{Si}$ : C, 59.24; $\mathrm{H}$, 4.85; N, 3.29. Found: C, 59.51; H, 4.98; N, 3.31.

10-(Dibutylboryl)-5-phenyl-1,9-di-p-toluoyldipyrromethane (7i). Following the general procedure (method A) described for $7 \mathbf{a}$, reaction of $\mathbf{6 a}(0.85 \mathrm{~g}, 1.83 \mathrm{mmol})$ in THF $(1.8$ $\mathrm{mL})$ with $\mathrm{MesMgBr}(3.66 \mathrm{~mL}, 3.66 \mathrm{mmol}, 1 \mathrm{M}$ solution in THF) followed by $2 \mathbf{a}(0.46 \mathrm{~g}, 2.01$ $\mathrm{mmol})$ in THF $(2 \mathrm{~mL})$ afforded a brown oil. After general workup, the brown residue was dissolved in a $5 \mathrm{~mL}$ solution of $\mathrm{Et}_{2} \mathrm{O} /$ hexanes (2:3). After a few minutes, a brownish yellow precipitate was observed. The resulting mixture was filtered on a Buchner funnel and washed 
with a small amount of hexanes $(3-5 \mathrm{~mL})$ to afford a yellow powder $(0.78 \mathrm{~g}, 73 \%)$ : $\mathrm{mp} 164{ }^{\circ} \mathrm{C}$ (dec.); ${ }^{1} \mathrm{H}$ NMR $\square 0.48 \square 0.71(\mathrm{~m}, 10 \mathrm{H}), 0.78 \square 1.13(\mathrm{~m}, 8 \mathrm{H}), 2.42(\mathrm{~s}, 3 \mathrm{H}), 2.48(\mathrm{~s}, 3 \mathrm{H}), 5.67$ (s, $1 \mathrm{H}), 6.03 \square 6.05(\mathrm{~m}, 1 \mathrm{H}), 6.45(\mathrm{~d}, J=4.0 \mathrm{~Hz}, 1 \mathrm{H}), 6.80 \square 6.82(\mathrm{~m}, 1 \mathrm{H}), 7.24 \square 7.29(\mathrm{~m}, 6 \mathrm{H}), 7.33$ $(\mathrm{d}, J=7.6 \mathrm{~Hz}, 2 \mathrm{H}), 7.37(\mathrm{~d}, J=7.6 \mathrm{~Hz}, 2 \mathrm{H}), 7.76(\mathrm{~d}, J=8.0 \mathrm{~Hz}, 2 \mathrm{H}), 8.12(\mathrm{~d}, J=8.0 \mathrm{~Hz}, 2 \mathrm{H})$,

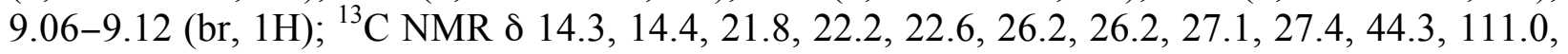
$117.9,119.1,120.0,127.7,127.9,128.6,129.1,129.1,130.10,130.12,131.0134 .4,135.9,140.1$, 140.3, 142.4, 145.7, 148.1, 177.1, 184.3; FABMS obsd 583.3536, calcd 583.3496 [(M+H) ${ }^{+}$, (M $\left.=\mathrm{C}_{39} \mathrm{H}_{43} \mathrm{BN}_{2} \mathrm{O}_{2}\right)$.

10-(Dibutylboryl)-1-(p-iodobenzoyl)-5-mesityl-9-p-toluoyldipyrromethane $(7 \mathbf{j})$. Following the general procedure (method A) described for 7a, reaction of $\mathbf{6 f} \square(1.23 \mathrm{~g}, 2.00$ $\mathrm{mmol})$ in THF $(2 \mathrm{~mL})$ with MesMgBr $(4.00 \mathrm{~mL}, 4.00 \mathrm{mmol}, 1 \mathrm{M}$ solution in THF) followed by 2 a $(0.50 \mathrm{~g}, 2.2 \mathrm{mmol})$ in THF $(4.4 \mathrm{~mL})$ afforded a brown oil. Chromatography [silica, $\mathrm{CH}_{2} \mathrm{Cl}_{2}$ /hexanes (1:2)] afforded a yellow solid (1.15 g, 78\%): $\mathrm{mp} \mathrm{80-82}{ }^{\circ} \mathrm{C}$ (dec.); ${ }^{1} \mathrm{H} \mathrm{NMR} \square$ $-0.08--0.03(\mathrm{~m}, 1 \mathrm{H}), 0.29-0.71(\mathrm{~m}, 7 \mathrm{H}), 0.72-0.97(\mathrm{~m}, 8 \mathrm{H}), 1.12-1.19(\mathrm{~m}, 2 \mathrm{H}), 2.15(\mathrm{~s}, 6 \mathrm{H})$, $2.27(\mathrm{~s}, 3 \mathrm{H}), 2.42(\mathrm{~s}, 3 \mathrm{H}), 5.94(\mathrm{~s}, 1 \mathrm{H}), 5.99-6.00(\mathrm{~m}, 1 \mathrm{H}), 6.45(\mathrm{~d}, J=4.4 \mathrm{~Hz}, 1 \mathrm{H}), 6.81-6.83$ $(\mathrm{m}, 1 \mathrm{H}), 6.85(\mathrm{~s}, 2 \mathrm{H}), 7.19(\mathrm{~d}, J=4.0 \mathrm{~Hz}, 1 \mathrm{H}), 7.27(\mathrm{~d}, J=8.0 \mathrm{~Hz}, 2 \mathrm{H}), 7.78(\mathrm{~d}, J=8.0 \mathrm{~Hz}$, 2H), 7.89(d, $J=8.0 \mathrm{~Hz}, 2 \mathrm{H}), 7.93(\mathrm{~d}, J=8.0 \mathrm{~Hz}, 2 \mathrm{H}), 9.03-9.11(\mathrm{br}, 1 \mathrm{H}) ;{ }^{13} \mathrm{C}$ NMR $\square 14.314 .4$, 20.9 , 21.2, 21.7, 21.8, 26.2, 27.2, 27.6, 40.2, 102.2, 11.2, 117.4, 120.1, 122.1, 129.2, 129.3, $129.9,130.6,130.8,131.0,133.5,135.5,135.9,137.2,137.5,138.6,139.2,142.4,150.1,175.5$, 184.1; FABMS obsd 737.2812, calcd 737.2775 [(M+H) $\left.{ }^{+}\right],\left(\mathrm{M}=\mathrm{C}_{41} \mathrm{H}_{46} \mathrm{BIN}_{2} \mathrm{O}_{2}\right)$; Anal. calcd for $\mathrm{C}_{41} \mathrm{H}_{46} \mathrm{BIN}_{2} \mathrm{O}_{2}$ : C, 66.86; H, 6.30; N, 3.80. Found: C, 67.24; H, 6.37; N, 3.74.

\section{0-(9-Borabicyclo[3.3.1] non-9-yl)-9-(p-methoxybenzoyl)-5-phenyl-1-p-}

toluoyldipyrromethane (7b). Following the general procedure (method B) described for 7a, reaction of $6 \mathbf{a}(0.92 \mathrm{~g}, 2.0 \mathrm{mmol})$ in THF $(2 \mathrm{~mL})$ with $\mathrm{MesMgBr}(4.0 \mathrm{~mL}, 4.00 \mathrm{mmol}, 1 \mathrm{M}$ solution in THF) followed by addition of $p$-anisoyl chloride $(0.30 \mathrm{~mL}, 2.2 \mathrm{mmol})$ in THF $(2.2$ $\mathrm{mL})$ afforded a yellow powder $(1.05 \mathrm{~g}, 88 \%)$ with satisfactory characterization data $\left(\mathrm{mp}\right.$, and ${ }^{1} \mathrm{H}$ NMR) as reported above. The elemental analysis for the sample assuming 0.5 molar equiv of water $\left(\mathrm{C}_{39} \mathrm{H}_{40} \mathrm{BN}_{2} \mathrm{O}_{3.5} ; \mathrm{C}, 77.61 ; \mathrm{H}, 6.68 ; \mathrm{N}, 4.64\right.$. Found: $\left.\mathrm{C}, 77.33 ; \mathrm{H}, 6.62 ; \mathrm{N}, 4.54\right)$ is in good agreement with the observed data.

\section{0-(9-Borabicyclo[3.3.1]non-9-yl)-9-(pentafluorobenzoyl)-5-phenyl-1-p-}

toluoyldipyrromethane (7c). Following the general procedure (method B) described for 7a, reaction of $6 \mathbf{a}(0.92 \mathrm{~g}, 2.0 \mathrm{mmol})$ in $\mathrm{THF}(2 \mathrm{~mL})$ with $\mathrm{MesMgBr}(4.0 \mathrm{~mL}, 4.0 \mathrm{mmol}, 1 \mathrm{M}$ solution in THF) followed by addition of pentafluorobenzoyl chloride $(0.32 \mathrm{~mL}, 2.2 \mathrm{mmol})$ in THF $(2.2 \mathrm{~mL})$ afforded a yellow powder $(1.21 \mathrm{~g}, 92 \%)$ with satisfactory characterization data (mp, ${ }^{1} \mathrm{H}$ NMR and elemental analysis) as reported above.

10-(9-Borabicyclo[3.3.1] non-9-yl)-1,9-di-p-toluoyldipyrromethane (7d). Following the general procedure (method B) described for $7 \mathbf{a}$, reaction of $\mathbf{6 b}(0.77 \mathrm{~g}, 2.0 \mathrm{mmol})$ in THF $(2$ $\mathrm{mL})$ with $\mathrm{Mes} \mathrm{MgBr}(4.0 \mathrm{~mL}, 4.0 \mathrm{mmol}, 1 \mathrm{M}$ solution in THF) followed by addition of $p$-toluoyl chloride $(0.29 \mathrm{~mL}, 2.2 \mathrm{mmol})$ in THF $(2.2 \mathrm{~mL})$ and the workup procedure described for $7 \mathbf{d}$ (method A) afforded a yellow powder $(0.90 \mathrm{~g}, 90 \%)$ with satisfactory characterization data (mp and ${ }^{1} \mathrm{H}$ NMR) as reported above. The elemental analysis for the sample assuming 0.5 molar equiv of water $\left(\mathrm{C}_{33} \mathrm{H}_{36} \mathrm{BN}_{2} \mathrm{O}_{2.5} ; \mathrm{C}, 77.49 ; \mathrm{H}, 7.09 ; \mathrm{N}, 5.48\right.$. Found: $\left.\mathrm{C}, 77.53 ; \mathrm{H}, 7.04 ; \mathrm{N}, 5.36\right)$ is in good agreement with the observed data.

10-(9-Borabicyclo[3.3.1]non-9-yl)-9-hexanoyl-1-p-toluoyldipyrromethane (7e). Following the general procedure (method B) described for 7a, reaction of $6 \mathbf{b}(0.77 \mathrm{~g}, 2.0 \mathrm{mmol})$ 
in THF ( $2 \mathrm{~mL})$ with $\operatorname{MesMgBr}(4.0 \mathrm{~mL}, 4.0 \mathrm{mmol}, 1 \mathrm{M}$ solution in THF) followed by addition of hexanoyl chloride $(0.30 \mathrm{~mL}, 2.2 \mathrm{mmol})$ in THF $(2.2 \mathrm{~mL})$ and the workup procedure for $7 \mathbf{e}$ (method A) afforded a yellow powder $(0.84 \mathrm{~g}, 87 \%)$ with satisfactory characterization data (mp and ${ }^{1} \mathrm{H}$ NMR) as reported above. The elemental analysis for the sample assuming 0.5 molar equiv of water $\left(\mathrm{C}_{31} \mathrm{H}_{40} \mathrm{BN}_{2} \mathrm{O}_{2.5} ; \mathrm{C}, 75.76 ; \mathrm{H}, 8.20 ; \mathrm{N}, 5.70\right.$. Found: $\left.\mathrm{C}, 75.38 ; \mathrm{H}, 8.03 ; \mathrm{N}, 5.65\right)$ is in good agreement with the observed data.

10-(9-Borabicyclo[3.3.1]non-9-yl)-9-(p-bromobenzoyl)-1-(p-methoxybenzoyl)-5-(pmethoxyphenyl)dipyrromethane (7f). Following the general procedure (method B) described for 7a, reaction of $\mathbf{6 c}(1.01 \mathrm{~g}, 2.0 \mathrm{mmol})$ in THF $(2 \mathrm{~mL})$ with $\operatorname{MesMgBr}(4.0 \mathrm{~mL}, 4.0 \mathrm{mmol}, 1 \mathrm{M}$ solution in THF) followed by addition of $p$-bromobenzoyl chloride $(0.48 \mathrm{~g}, 2.2 \mathrm{mmol})$ in THF $(2.2 \mathrm{~mL})$ afforded a yellow powder $(1.08 \mathrm{~g}, 79 \%)$ with satisfactory characterization data $\left(\mathrm{mp},{ }^{1} \mathrm{H}\right.$ NMR and elemental analysis) as reported above.

10-(9-Borabicyclo[3.3.1]non-9-yl)-1-p-hexanoyl-5-pentyl-9-p-toluoyldipyrromethane (7g). Following the general procedure (method B) described for $7 \mathbf{a}$, reaction of $\mathbf{6 d}(0.87 \mathrm{~g}, 2.0$ mmol) in THF ( $2 \mathrm{~mL})$ with $\mathrm{Mes} \mathrm{MgBr}(4.0 \mathrm{~mL}, 4.0 \mathrm{mmol}, 1 \mathrm{M}$ solution in THF) followed by addition of $p$-toluoyl chloride $(0.30 \mathrm{~mL}, 2.2 \mathrm{mmol})$ in THF $(2.2 \mathrm{~mL})$ afforded an off-white powder $(0.70 \mathrm{~g}, 64 \%)$ with satisfactory characterization data $\left(\mathrm{mp},{ }^{1} \mathrm{H}\right.$ NMR and elemental analysis) as reported above.

\section{0-(9-Borabicyclo[3.3.1]non-9-yl)-1-(p-bromobenzoyl)-9-(p-iodobenzoyl)-5-[p-} (trimethylsilylethynyl)phenyl]dipyrromethane $(\mathbf{7 h})$. Following the general procedure (method B) described for 7a, reaction of $\mathbf{6 e}(1.24 \mathrm{~g}$, $2.0 \mathrm{mmol})$ in THF $(2 \mathrm{~mL})$ with $\mathrm{MesMgBr}$ ( $4.0 \mathrm{~mL}, 4.0 \mathrm{mmol}, 1 \mathrm{M}$ solution in THF) followed by addition of $p$-iodobenzoyl chloride $(0.57$ $\mathrm{g}, 2.2 \mathrm{mmol})$ in THF $(2.2 \mathrm{~mL})$ afforded a yellow-orange powder $(1.17 \mathrm{~g}, 69 \%)$ with satisfactory characterization data (mp, ${ }^{1} \mathrm{H}$ NMR and elemental analysis) as reported above.

10-(Dibutylboryl)-5-phenyl-1,9-di-p-toluoyldipyrromethane (7i). Following the general procedure (method B) described for 7a, reaction of $6 \mathrm{a} \square(0.93 \mathrm{~g}, 2.0 \mathrm{mmol}$ ) in THF (2 $\mathrm{mL})$ with $\mathrm{Mes} \mathrm{MgBr}(4.0 \mathrm{~mL}, 4.0 \mathrm{mmol}, 1 \mathrm{M}$ solution in THF) followed by addition of $p$-toluoyl chloride $(0.30 \mathrm{~mL}, 2.2 \mathrm{mmol})$ in THF $(2.2 \mathrm{~mL})$ afforded an off-white powder $(0.91 \mathrm{~g}, 78 \%)$ with satisfactory characterization data (mp, ${ }^{1} \mathrm{H}$ NMR and elemental analysis) as reported above.

10-(Dibutylboryl)-1-( $p$-iodobenzoyl)-5-mesityl-9-p-toluoyldipyrromethane (7j). Following the general procedure (method B) described for 7a, reaction of $\mathbf{6 f} \square(1.48 \mathrm{~g}, 2.0 \mathrm{mmol}$ ) in THF $(2 \mathrm{~mL})$ with $\operatorname{MesMgBr}(4.0 \mathrm{~mL}, 4.0 \mathrm{mmol}, 1 \mathrm{M}$ solution in THF) followed by addition of $p$-toluoyl chloride $(0.30 \mathrm{~mL}, 2.2 \mathrm{mmol})$ in THF $(2.2 \mathrm{~mL})$ afforded a yellow powder $(1.14 \mathrm{~g}$, $77 \%$ ) with satisfactory characterization data (mp, ${ }^{1} \mathrm{H}$ NMR and FABMS) as reported above.

Removal of Solvent of Inclusion in Samples of 1,9-Diacyldipyrromethane-BR Complexes. A number of samples of 1,9-diacyldipyrromethane-BR $\mathrm{B}_{2}$ complexes were found upon ${ }^{1} \mathrm{H}$ NMR analysis to include solvent (e.g., diethyl ether, THF) in the crystal lattice. To remove the solvent, the solid sample was dissolved in a minimum amount of $\mathrm{CH}_{2} \mathrm{Cl}_{2}$. The solution was treated with hexanes. The solution was concentrated to dryness in vacuo. The residue in the evaporation flask was triturated with hexanes 2-3 times and the solvents were again removed in vacuo. The resulting ${ }^{1} \mathrm{H}$ NMR spectrum typically showed the absence of peaks due to the ethereal solvent, but did show the presence of a peak due to hexane or $\mathrm{CH}_{2} \mathrm{Cl}_{2}$. Any peak due to water was not unambiguously ascribable to that from solvent of inclusion; however, a number of samples upon elemental analysis afforded satisfactory results upon assuming the presence of 1 molecule of water per two molecules of 1,9-diacyldipyrromethane-BR $\mathrm{B}_{2}$ complex. 
Alternative Procedure for 9-Acylation Using HMDS. A suspension of 6a ( $0.46 \mathrm{~g}, 1.0$ $\mathrm{mmol})$ in THF $(1 \mathrm{~mL})$ and HMDS $(0.21 \mathrm{~mL}, 1.0 \mathrm{mmol})$ was treated with $\operatorname{MesMgBr}(2.0 \mathrm{~mL}, 2.0$ mmol, $1 \mathrm{M}$ solution in THF). The mixture was stirred at room temperature for $5 \mathrm{~min}$. Then a solution of $2 \mathrm{a}(0.25 \mathrm{~g}, 1.10 \mathrm{mmol})$ in THF $(1.1 \mathrm{~mL})$ was added. The mixture was stirred at room temperature for $10 \mathrm{~min}$. The mixture was quenched by addition of a half-saturated aqueous solution of $\mathrm{NH}_{4} \mathrm{Cl}(10 \mathrm{~mL})$. $\mathrm{Et}_{2} \mathrm{O}(20 \mathrm{~mL})$ was added. The organic layer was separated and washed with a saturated aqueous solution of $\mathrm{NaHCO}_{3}(20 \mathrm{~mL})$ followed by brine $(10 \mathrm{~mL})$. The organic layer was dried $\left(\mathrm{Na}_{2} \mathrm{SO}_{4}\right)$ and concentrated to dryness under vacuum. The resulting brown residue was treated with a small amount of $\mathrm{Et}_{2} \mathrm{O}(\square 2-4 \mathrm{~mL})$, affording a suspension consisting of a brown solution and a bright yellow powder. The resulting mixture was filtered on a Buchner funnel and washed with a small amount of hexanes $(\square 5-10 \mathrm{~mL})$. The filtered material $(0.48 \mathrm{~g}, 83 \%$, nearly pure, see Note 1$)$ was treated with $\mathrm{CH}_{2} \mathrm{Cl}_{2} /$ hexanes $(10 \mathrm{~mL}, 1: 2)$ affording a suspension. The suspension was concentrated to one-fifth of the starting volume. The resulting mixture was filtered on a Buchner funnel. The precipitate was washed with hexanes to afford the title compound ( $0.44 \mathrm{~g}, 76 \%)$ : $\mathrm{mp} 190 \square 192{ }^{\circ} \mathrm{C}(\mathrm{dec}.) ;{ }^{1} \mathrm{H}$ NMR $\square 0.66 \square 0.74(\mathrm{~m}, 2 \mathrm{H}), 1.67 \square 2.29$ $(\mathrm{m}, 12 \mathrm{H}), 2.42(\mathrm{~s}, 3 \mathrm{H}), 2.48(\mathrm{~s}, 3 \mathrm{H}), 6.00 \square 6.02(\mathrm{~m}, 1 \mathrm{H}), 6.10(\mathrm{~s}, 1 \mathrm{H}), 6.46(\mathrm{~d}, J=4.4 \mathrm{~Hz}, 1 \mathrm{H})$, $6.80 \square 6.82(\mathrm{~m}, 1 \mathrm{H}), 7.18(\mathrm{~d}, J=8.0 \mathrm{~Hz}, 2 \mathrm{H}), 7.26 \square 7.38(\mathrm{~m}, 8 \mathrm{H}), 7.77(\mathrm{~d}, J=8.0 \mathrm{~Hz}, 2 \mathrm{H}), 8.13$ (d, $J=8.0 \mathrm{~Hz}, 2 \mathrm{H}), 9.10 \square 9.16(\mathrm{br}, 1 \mathrm{H})$. FABMS obsd 579.3183 , calcd $579.3226\left[(\mathrm{M}+\mathrm{H})^{+}\right],(\mathrm{M}$ $=\mathrm{C}_{39} \mathrm{H}_{39} \mathrm{BN}_{2} \mathrm{O}_{2}$ ). The elemental analysis for the sample assuming 0.5 molar equiv of water $\left(\mathrm{C}_{39} \mathrm{H}_{40} \mathrm{BN}_{2} \mathrm{O}_{2.5} ; \mathrm{C}, 79.72 ; \mathrm{H}, 6.86 ; \mathrm{N}, 4.77\right.$. Found: $\left.\mathrm{C}, 80.13 ; \mathrm{H}, 6.75 ; \mathrm{N}, 4.85\right)$ is in good agreement with the observed data.

Note 1: The ${ }^{1} \mathrm{H}$ NMR spectrum showed the presence of one molecule of $\mathrm{Et}_{2} \mathrm{O}$ per two molecules of the title compound: ${ }^{1} \mathrm{H}$ NMR $\square 0.67 \square 0.73(\mathrm{~m}, 2 \mathrm{H}), 1.21(\mathrm{t}, J=6.8 \mathrm{~Hz}, 6 \mathrm{H}$; derived from $\left.\mathrm{Et}_{2} \mathrm{O}\right), 1.67 \square 2.27(\mathrm{~m}, 12 \mathrm{H}), 2.42(\mathrm{~s}, 3 \mathrm{H}), 2.48(\mathrm{~s}, 3 \mathrm{H}), 3.48(\mathrm{q}, J=6.8 \mathrm{~Hz}, 4 \mathrm{H}$; derived from $\left.\mathrm{Et}_{2} \mathrm{O}\right), 6.00 \square 6.02(\mathrm{~m}, 1 \mathrm{H}), 6.10(\mathrm{~s}, 1 \mathrm{H}), 6.46(\mathrm{~d}, J=4.0 \mathrm{~Hz}, 1 \mathrm{H}), 6.80 \square 6.82(\mathrm{~m}, 1 \mathrm{H}), 7.18(\mathrm{~d}, J=$ $8.0 \mathrm{~Hz}, 2 \mathrm{H}), 7.26 \square 7.38(\mathrm{~m}, 8 \mathrm{H}), 7.77(\mathrm{~d}, J=8.0 \mathrm{~Hz}, 2 \mathrm{H}), 8.13(\mathrm{~d}, J=8.0 \mathrm{~Hz}, 2 \mathrm{H}), 9.11 \square 9.17$ (br, $1 \mathrm{H})$. The elemental analysis calculated for $\mathrm{C}_{41} \mathrm{H}_{44} \mathrm{BN}_{2} \mathrm{O}_{2.5}(\mathrm{C}, 79.99 ; \mathrm{H}, 7.20 ; \mathrm{N}, 4.55$. Found: C, 79.81; H, 7.06; N, 4.69 ) is in good agreement with the observed data. 


\section{X-ray Structural Determination for $7 \mathrm{~b}-\mathrm{BBN}$.}

Data Collection. A crystal of $\mathbf{7 b}-\mathbf{B B N}$ was mounted on the end of a glass fiber with a small amount of silicone grease, and transferred to the diffractometer. All X-ray measurements were made on an Enraf-Nonius CAD4-MACH diffractometer at $-125{ }^{\circ} \mathrm{C}$. The unit cell dimensions were determined by a symmetry constrained fit of 25 well centered reflections and their Friedel pairs with $30^{\circ}<2(\square)<36^{\circ}$. A quadrant of unique data was collected using the $\square$ scan mode in non-bisecting geometry. The adoption of a non-bisecting scan mode was accomplished by offsetting psi by 20.00 for each data point collected. This was done to minimize the interaction of the goniometer head with the cold stream. Three standard reflections were measured every 4800 seconds of X-ray exposure time. Scaling the data was accomplished using a 5 point smoothed curved routine fit to the intensity check reflections. The intensity data was corrected for Lorentz and polarization effects. No absorption correction was made.

Structure Solution and Refinement. The data were reduced using routines from the NRCVAX set of programs. The structure was solved using SIR92. All non-hydrogen atoms were recovered from the initial E-map. The hydrogen atom positions were introduced at idealized positions. During the intermediate cycles of refinement, the methyl hydrogens were refined as a group. After these refinements converged, these hydrogens were treated the same as the other hydrogen atoms which were allowed to ride on their respective parent carbon or nitrogen atoms. All non-hydrogen atoms were refined anisotropically. The calculated structure factors were fit to the data using full matrix least-squares based on F. The calculated structure factors included corrections for anomalous dispersion from the usual tabulation (International Tables for X-ray Crystallography, Vol. IV, (1974), Table 2.3.1, Kynoch Press, Birmingham, England). A secondary extinction correction was included in the final refinements.

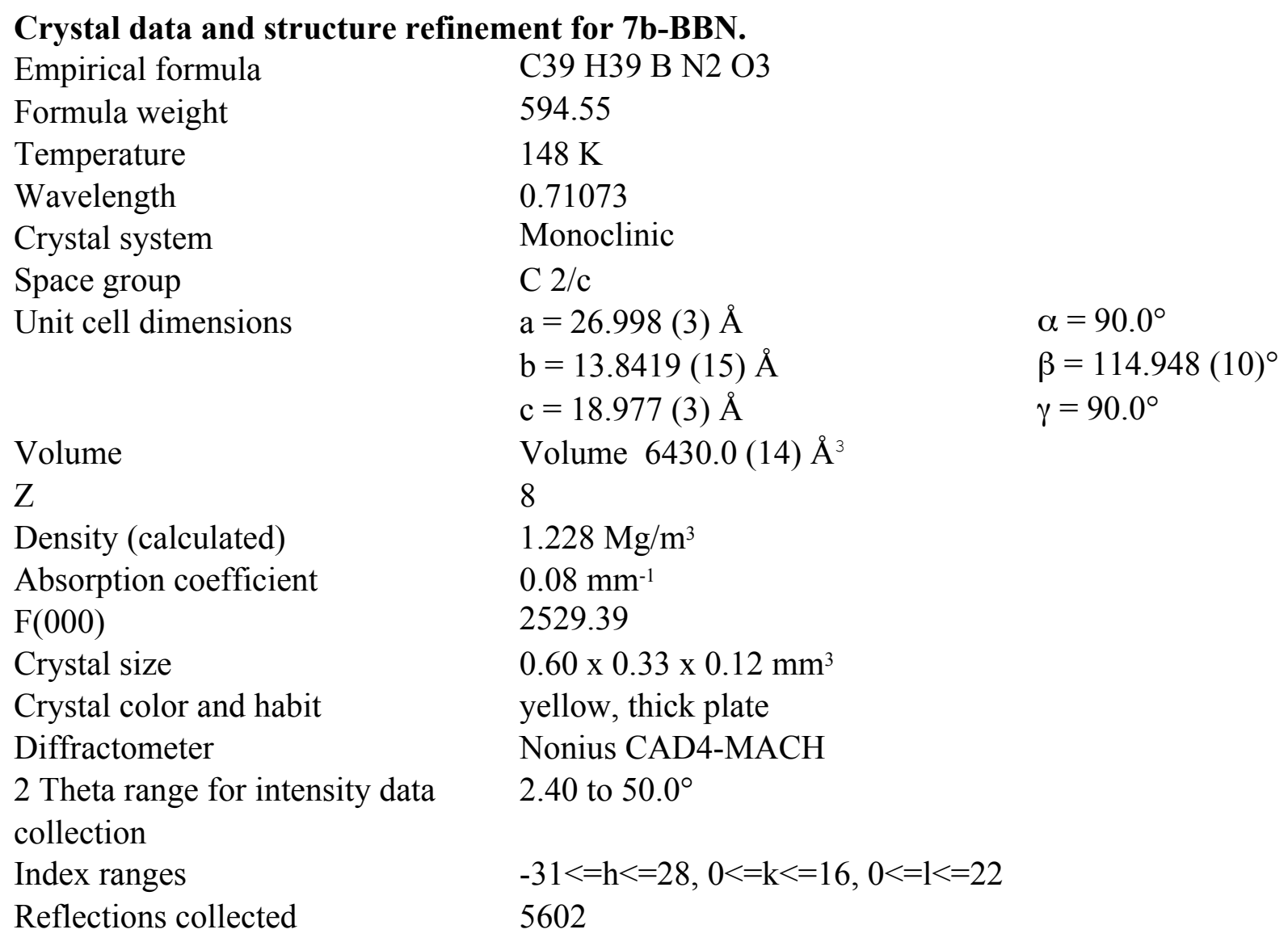


Independent reflections

Observed reflections (I >

1sigma(I))

Absorption correction

Max. and min. transmission

Solution method

Refinement method

Data / parameters

Goodness-of-fit

Final $\mathrm{R}$ indices (based on $\mathrm{F}$ )

$\mathrm{R}$ indices (all data)

Largest diff. peak and hole
5602

3689

None

NA

Direct methods (SIR 92)

Full matrix least-squares based on $\mathrm{F}$ $3683 / 407$

1.39

$\mathrm{R}=0.047, \mathrm{Rw}=0.049$

$\mathrm{R}=0.100, \mathrm{Rw}=0.050$

0.190 and -0.190 e. $\AA^{-3}$ 
Atomic coordinates and equivalent isotropic displacement parameters $\left(\AA^{2}\right)$ for $7 \mathrm{~b}-\mathrm{BBN}$.

\begin{tabular}{|c|c|c|c|}
\hline Name & $\mathbf{x}$ & $\mathbf{y}$ & $\mathbf{Z}$ \\
\hline B1 & $.51959(10)$ & $.31560(21)$ & $.55959(15)$ \\
\hline $\mathrm{O} 1$ & $.46041(6)$ & $.33347(12)$ & $.55319(9)$ \\
\hline N1 & $.50157(6)$ & $.28133(13)$ & $.47172(10)$ \\
\hline $\mathrm{C} 1$ & $.52058(8)$ & $.25416(17)$ & $.42007(12)$ \\
\hline $\mathrm{C} 2$ & $.47617(9)$ & $.24509(20)$ & $.34631(13)$ \\
\hline $\mathrm{C} 3$ & $.42886(8)$ & $.26664(18)$ & $.35366(13)$ \\
\hline $\mathrm{C} 4$ & $.44468(8)$ & $.28903(16)$ & $.43180(13)$ \\
\hline $\mathrm{C} 5$ & $.57888(8)$ & $.22613(17)$ & $.43851(12)$ \\
\hline C6 & $.60596(8)$ & $.29521(17)$ & $.40453(12)$ \\
\hline $\mathrm{N} 2$ & $.66111(7)$ & $.29519(13)$ & $.42953(10)$ \\
\hline $\mathrm{C} 7$ & $.58515(9)$ & $.36555(18)$ & $.34807(13)$ \\
\hline $\mathrm{C} 8$ & $.62907(9)$ & .40715(17) & $.33799(13)$ \\
\hline C9 & $.67665(8)$ & $.36230(16)$ & $.38938(12)$ \\
\hline $\mathrm{C} 10$ & $.73423(9)$ & $.37739(16)$ & $.40924(13)$ \\
\hline $\mathrm{O} 2$ & $.76895(6)$ & $.34342(12)$ & $.47060(9)$ \\
\hline $\mathrm{C} 11$ & $.75226(8)$ & $.43546(16)$ & $.35870(13)$ \\
\hline $\mathrm{C} 12$ & $.72348(9)$ & $.44268(19)$ & $.27865(14)$ \\
\hline C13 & $.74396(9)$ & 49473(19) & $.23443(14)$ \\
\hline C14 & $.79383(9)$ & $.54145(18)$ & $.27029(16)$ \\
\hline $\mathrm{C} 15$ & $.82366(9)$ & $.53312(18)$ & $.34998(16)$ \\
\hline $\mathrm{C} 16$ & $.80331(9)$ & .48096(18) & $.39340(14)$ \\
\hline $\mathrm{O} 3$ & $.81686(7)$ & $.59660(14)$ & $.23244(11)$ \\
\hline $\mathrm{C} 17$ & $.78662(11)$ & $.60865(23)$ & $.15070(18)$ \\
\hline C18 & $.57707(8)$ & $.12109(17)$ & $.41293(13)$ \\
\hline C19 & $.58145(9)$ & $.09430(18)$ & $.34551(14)$ \\
\hline $\mathrm{C} 20$ & $.57404(10)$ & $-.00208(21)$ & $.32131(15)$ \\
\hline $\mathrm{C} 21$ & $.56299(10)$ & $-.07121(19)$ & $.36492(17)$ \\
\hline $\mathrm{C} 22$ & $.55938(9)$ & $-.04545(20)$ & $.43252(16)$ \\
\hline $\mathrm{C} 23$ & $.56588(9)$ & $.04966(19)$ & $.45627(14)$ \\
\hline $\mathrm{C} 24$ & $.42300(9)$ & $.31760(17)$ & $.48345(14)$ \\
\hline $\mathrm{C} 25$ & $.36538(9)$ & $.32959(17)$ & $.46805(14)$ \\
\hline $\mathrm{C} 26$ & $.32440(9)$ & $.28413(19)$ & $.40468(15)$ \\
\hline $\mathrm{C} 27$ & $.27006(10)$ & $.29339(22)$ & $.39175(17)$ \\
\hline $\mathrm{C} 28$ & $.25519(10)$ & $.34827(20)$ & $.44066(18)$ \\
\hline $\mathrm{C} 29$ & $.29604(11)$ & $.39399(21)$ & $.50317(18)$ \\
\hline $\mathrm{C} 30$ & $.35080(10)$ & $.38489(20)$ & $.51784(17)$ \\
\hline C31 & $.19568(12)$ & $.35817(24)$ & $.42591(21)$ \\
\hline $\mathrm{C} 32$ & $.55328(9)$ & $.41443(17)$ & $.58238(13)$ \\
\hline $\mathrm{C} 33$ & $.55036(10)$ & $.45472(19)$ & $.65583(16)$ \\
\hline C34 & $.56782(11)$ & $.38326(21)$ & $.72410(15)$ \\
\hline $\mathrm{C} 35$ & $.54506(10)$ & $.28049(19)$ & $.70148(14)$ \\
\hline $\mathrm{C} 36$ & $.55007(9)$ & $.24035(17)$ & $.62892(13)$ \\
\hline C37 & $.60983(9)$ & $.22393(17)$ & $.64369(13)$ \\
\hline $\mathrm{C} 38$ & $.64327(9)$ & $.31521(19)$ & $.64695(14)$ \\
\hline
\end{tabular}




$\begin{array}{llll}\mathrm{C} 39 & .61191(9) & .39682(18) & .59113(13) \\ \mathrm{H} 2 & .47874(0) & .22662(0) & .29919(0) \\ \mathrm{H} 2 \mathrm{n} & .68320(0) & .25819(0) & .46545(0) \\ \mathrm{H} 3 & .39238(0) & .26662(0) & .31322(0) \\ \mathrm{H} 5 & .59903(0) & .22756(0) & .49399(0) \\ \mathrm{H} 7 & .54734(0) & .38292(0) & .32098(0) \\ \mathrm{H} 8 & .62690(0) & .45727(0) & .30183(0) \\ \mathrm{H} 12 & .68850(0) & .41180(0) & .25358(0) \\ \mathrm{H} 13 & .72402(0) & .49785(0) & .17897(0) \\ \mathrm{H} 15 & .85855(0) & .56437(0) & .37473(0) \\ \mathrm{H} 16 & .82438(0) & .47509(0) & .44850(0) \\ \mathrm{H} 17 \mathrm{a} & .80714(36) & .64796(112) & .13083(23) \\ \mathrm{H} 17 \mathrm{~b} & .75240(34) & .63949(122) & .14041(18) \\ \mathrm{H} 17 \mathrm{c} & .77996(64) & .54659(31) & .12581(18) \\ \mathrm{H} 19 & .58929(0) & .14238(0) & .31519(0) \\ \mathrm{H} 20 & .57665(0) & -.02022(0) & .27416(0) \\ \mathrm{H} 21 & .55795(0) & .13731(0) & .34802(0) \\ \mathrm{H} 22 & .55234(0) & . .09401(0) & .46325(0) \\ \mathrm{H} 23 & .56282(0) & .06746(0) & .50317(0) \\ \mathrm{H} 26 & .33406(0) & .24669(0) & .36976(0) \\ \mathrm{H} 27 & .24204(0) & .26106(0) & .34854(0) \\ \mathrm{H} 29 & .28598(0) & .43310(0) & .53685(0) \\ \mathrm{H} 30 & .37862(0) & .41628(0) & .56187(0) \\ \mathrm{H} 31 \mathrm{a} & .18944(23) & .42165(58) & .44088(107) \\ \mathrm{H} 31 \mathrm{~b} & .18694(25) & .31074(95) & .45582(92) \\ \mathrm{H} 31 \mathrm{c} & .17297(11) & .34845(141) & .37165(28) \\ \mathrm{H} 32 & .53565(0) & .45902(0) & .54043(0) \\ \mathrm{H} 33 \mathrm{a} & .57302(0) & .51119(0) & .67225(0) \\ \mathrm{H} 33 \mathrm{~b} & .51308(0) & .47226(0) & .64257(0) \\ \mathrm{H} 34 \mathrm{a} & .60696(0) & .37903(0) & .74645(0) \\ \mathrm{H} 34 \mathrm{~b} & .55636(0) & .40804(0) & .76211(0) \\ \mathrm{H} 35 \mathrm{a} & .50701(0) & .28210(0) & .69084(0) \\ \mathrm{H} 35 \mathrm{~b} & .56403(0) & .23790(0) & .74442(0) \\ \mathrm{H} 36 & .53076(0) & .18013(0) & .61433(0) \\ \mathrm{H} 37 \mathrm{a} & .62765(0) & .19064(0) & .69235(0) \\ \mathrm{H} 37 \mathrm{~b} & .61017(0) & .18400(0) & .60253(0) \\ \mathrm{H} 38 \mathrm{a} & .65677(0) & .34028(0) & .69889(0) \\ \mathrm{H} 38 \mathrm{~b} & .67347(0) & .29702(0) & .63567(0) \\ \mathrm{H} 39 \mathrm{a} & .63227(0) & .45552(0) & .60936(0) \\ \mathrm{H} 39 \mathrm{~b} & .60994(0) & .38113(0) & .54073(0)\end{array}$


Bond lengths $[\AA ̊]$ for $7 \mathrm{~b}-\mathrm{BBN}$.

\begin{tabular}{|c|c|c|c|}
\hline B1-O1 & $1.570(3)$ & C19-H19 & $.960(3)$ \\
\hline B1-N1 & $1.599(3)$ & C20-C21 & $1.378(4)$ \\
\hline B1-C32 & $1.598(4)$ & $\mathrm{C} 20-\mathrm{H} 20$ & $.960(3)$ \\
\hline B1-C36 & $1.606(4)$ & C21-C22 & $1.374(4)$ \\
\hline $\mathrm{O} 1-\mathrm{C} 24$ & $1.301(3)$ & C21-H21 & $.960(3)$ \\
\hline N1-C1 & $1.338(3)$ & C22-C23 & $1.379(4)$ \\
\hline $\mathrm{N} 1-\mathrm{C} 4$ & $1.401(3)$ & C22-H22 & $.960(3)$ \\
\hline $\mathrm{C} 1-\mathrm{C} 2$ & $1.413(3)$ & $\mathrm{C} 23-\mathrm{H} 23$ & $.960(3)$ \\
\hline $\mathrm{C} 1-\mathrm{C} 5$ & $1.512(3)$ & C24-C25 & $1.466(3)$ \\
\hline $\mathrm{C} 2-\mathrm{C} 3$ & $1.375(3)$ & C25-C26 & $1.394(4)$ \\
\hline $\mathrm{C} 2-\mathrm{H} 2$ & $.960(2)$ & $\mathrm{C} 25-\mathrm{C} 30$ & $1.395(4)$ \\
\hline C3-C4 & $1.394(3)$ & C26-C27 & $1.388(4)$ \\
\hline C3-H3 & $.960(2)$ & $\mathrm{C} 26-\mathrm{H} 26$ & $.960(3)$ \\
\hline C4-C24 & $1.394(3)$ & C27-C28 & $1.383(4)$ \\
\hline C5-C6 & $1.504(3)$ & $\mathrm{C} 27-\mathrm{H} 27$ & $.960(3)$ \\
\hline C5-C18 & $1.527(3)$ & C28-C29 & $1.386(4)$ \\
\hline C5-H5 & $.960(2)$ & C28-C31 & $1.516(4)$ \\
\hline C6-N2 & $1.359(3)$ & C29-C30 & $1.390(4)$ \\
\hline C6-C7 & $1.380(3)$ & C29-H29 & $.960(3)$ \\
\hline N2-C9 & $1.375(3)$ & C30-H30 & $.960(3)$ \\
\hline $\mathrm{N} 2-\mathrm{H} 2 \mathrm{n}$ & $.8600(17)$ & C31-H31a & $.960(11)$ \\
\hline C7-C8 & $1.402(3)$ & $\mathrm{C} 31-\mathrm{H} 31 \mathrm{~b}$ & $.960(16)$ \\
\hline C7-H7 & $.960(2)$ & C31-H31c & $.960(6)$ \\
\hline C8-C9 & $1.389(3)$ & C32-C33 & $1.534(4)$ \\
\hline C8-H8 & $.960(2)$ & C32-C39 & $1.540(3)$ \\
\hline C9-C10 & $1.452(3)$ & C32-H32 & $.960(2)$ \\
\hline C10-O2 & $1.239(3)$ & C33-C34 & $1.537(4)$ \\
\hline C10-C11 & $1.482(3)$ & C33-H33a & $.960(3)$ \\
\hline C11-C12 & $1.388(3)$ & C33-H33b & $.960(3)$ \\
\hline C11-C16 & $1.402(3)$ & C34-C35 & $1.537(4)$ \\
\hline C12-C13 & $1.386(3)$ & C34-H34a & $.960(3)$ \\
\hline C12-H12 & $.960(2)$ & C34-H34b & $.960(3)$ \\
\hline C13-C14 & $1.386(4)$ & C35-C36 & $1.543(3)$ \\
\hline C13-H13 & $.960(2)$ & C35-H35a & $.960(3)$ \\
\hline C14-C15 & $1.386(4)$ & C35-H35b & $.960(3)$ \\
\hline C14-O3 & $1.365(3)$ & C36-C37 & $1.533(3)$ \\
\hline C15-C16 & $1.373(4)$ & C36-H36 & $.960(2)$ \\
\hline C15-H15 & $.960(2)$ & C37-C38 & $1.539(4)$ \\
\hline C16-H16 & $.960(2)$ & C37-H37a & $.960(2)$ \\
\hline O3-C17 & $1.425(4)$ & C37-H37b & $.960(2)$ \\
\hline C17-H17a & $.960(12)$ & C38-C39 & $1.536(4)$ \\
\hline C17-H17b & $.960(11)$ & C38-H38a & $.960(3)$ \\
\hline $\mathrm{C} 17-\mathrm{H} 17 \mathrm{c}$ & $.960(5)$ & C38-H38b & $.960(3)$ \\
\hline C18-C19 & $1.384(3)$ & C39-H39a & $.960(2)$ \\
\hline C18-C23 & $1.398(3)$ & C39-H39b & $.960(2)$ \\
\hline C19-C20 & $1.398(4)$ & & \\
\hline
\end{tabular}


Bond angles [deg] for $7 \mathrm{~b}-\mathrm{BBN}$.

\begin{tabular}{|c|c|c|c|}
\hline O1-B1-N1 & $96.54(16)$ & C22-C21-H21 & $120.2(3)$ \\
\hline O1-B1-C32 & $109.3(2)$ & $\mathrm{C} 21-\mathrm{C} 22-\mathrm{C} 23$ & $120.2(3)$ \\
\hline O1-B1-C36 & $108.58(19)$ & $\mathrm{C} 21-\mathrm{C} 22-\mathrm{H} 22$ & $119.7(3)$ \\
\hline N1-B1-C32 & $115.1(2)$ & $\mathrm{C} 23-\mathrm{C} 22-\mathrm{H} 22$ & $120.0(3)$ \\
\hline N1-B1-C36 & $119.1(2)$ & $\mathrm{C} 18-\mathrm{C} 23-\mathrm{C} 22$ & $120.8(2)$ \\
\hline C32-B1-C36 & $107.28(19)$ & C18-C23-H23 & $119.2(3)$ \\
\hline B1-O1-C24 & $112.35(18)$ & $\mathrm{C} 22-\mathrm{C} 23-\mathrm{H} 23$ & $120.0(3)$ \\
\hline B1-N1-C1 & $143.62(18)$ & O1-C24-C4 & $112.77(20)$ \\
\hline B1-N1-C4 & $108.86(17)$ & $\mathrm{O} 1-\mathrm{C} 24-\mathrm{C} 25$ & $119.1(2)$ \\
\hline C1-N1-C4 & 107.29(17) & C4-C24-C25 & $128.1(2)$ \\
\hline $\mathrm{N} 1-\mathrm{C} 1-\mathrm{C} 2$ & 108.86(19) & $\mathrm{C} 24-\mathrm{C} 25-\mathrm{C} 26$ & $120.7(2)$ \\
\hline $\mathrm{N} 1-\mathrm{C} 1-\mathrm{C} 5$ & 125.90(19) & $\mathrm{C} 24-\mathrm{C} 25-\mathrm{C} 30$ & $120.4(2)$ \\
\hline $\mathrm{C} 2-\mathrm{C} 1-\mathrm{C} 5$ & $124.9(2)$ & C26-C25-C30 & $118.9(2)$ \\
\hline $\mathrm{C} 1-\mathrm{C} 2-\mathrm{C} 3$ & $108.5(2)$ & $\mathrm{C} 25-\mathrm{C} 26-\mathrm{C} 27$ & $120.4(2)$ \\
\hline $\mathrm{C} 1-\mathrm{C} 2-\mathrm{H} 2$ & $125.6(2)$ & $\mathrm{C} 25-\mathrm{C} 26-\mathrm{H} 26$ & $119.4(2)$ \\
\hline $\mathrm{C} 3-\mathrm{C} 2-\mathrm{H} 2$ & $126.0(2)$ & $\mathrm{C} 27-\mathrm{C} 26-\mathrm{H} 26$ & $120.2(3)$ \\
\hline $\mathrm{C} 2-\mathrm{C} 3-\mathrm{C} 4$ & 105.99(19) & $\mathrm{C} 26-\mathrm{C} 27-\mathrm{C} 28$ & $121.1(3)$ \\
\hline C2-C3-H3 & $127.1(2)$ & $\mathrm{C} 26-\mathrm{C} 27-\mathrm{H} 27$ & $120.1(3)$ \\
\hline C4-C3-H3 & $126.9(2)$ & C28-C27-H27 & $118.8(3)$ \\
\hline N1-C4-C3 & $109.40(19)$ & $\mathrm{C} 27-\mathrm{C} 28-\mathrm{C} 29$ & $118.3(2)$ \\
\hline N1-C4-C24 & 109.34(19) & C27-C28-C31 & $120.7(3)$ \\
\hline C3-C4-C24 & $141.3(2)$ & C29-C28-C31 & $121.0(3)$ \\
\hline C1-C5-C6 & 112.61(19) & C28-C29-C30 & $121.7(3)$ \\
\hline C1-C5-C18 & $106.27(18)$ & C28-C29-H29 & $118.7(3)$ \\
\hline C1-C5-H5 & $107.55(19)$ & С30-C29-H29 & $119.6(3)$ \\
\hline C6-C5-C18 & 115.17(19) & $\mathrm{C} 25-\mathrm{C} 30-\mathrm{C} 29$ & $119.6(2)$ \\
\hline C6-C5-H5 & 107.70(19) & C25-C30-H30 & $119.8(2)$ \\
\hline C18-C5-H5 & $107.2(2)$ & C29-C30-H30 & $120.6(3)$ \\
\hline C5-C6-N2 & $120.62(19)$ & C28-C31-H31a & $109.4(5)$ \\
\hline $\mathrm{C} 5-\mathrm{C} 6-\mathrm{C} 7$ & $132.00(20)$ & C28-C31-H31b & $109.5(5)$ \\
\hline N2-C6-C7 & $107.38(20)$ & C28-C31-H31c & $109.5(4)$ \\
\hline C6-N2-C9 & $110.61(18)$ & H31a-C31-H31b & $109.5(15)$ \\
\hline C6-N2-H2n & $124.6(2)$ & H31a-C31-H31c & $109.5(14)$ \\
\hline C9-N2-H2n & $124.75(19)$ & $\mathrm{H} 31 \mathrm{~b}-\mathrm{C} 31-\mathrm{H} 31 \mathrm{c}$ & $109.5(12)$ \\
\hline C6-C7-C8 & $107.76(19)$ & B1-C32-C33 & $108.4(2)$ \\
\hline C6-C7-H7 & $125.8(2)$ & B1-C32-C39 & $109.61(20)$ \\
\hline C8-C7-H7 & $126.4(2)$ & B1-C32-H32 & $107.4(2)$ \\
\hline C7-C8-C9 & $107.8(2)$ & C33-C32-C39 & $113.84(20)$ \\
\hline C7-C8-H8 & $126.5(2)$ & C33-C32-H32 & $108.8(2)$ \\
\hline C9-C8-H8 & $125.7(2)$ & C39-C32-H32 & $108.6(2)$ \\
\hline $\mathrm{N} 2-\mathrm{C} 9-\mathrm{C} 8$ & $106.43(19)$ & C32-C33-C34 & $114.6(2)$ \\
\hline N2-C9-C10 & 119.81(19) & C32-C33-H33a & $108.7(2)$ \\
\hline C8-C9-C10 & $133.6(2)$ & C32-C33-H33b & $107.7(2)$ \\
\hline C9-C10-O2 & $119.5(2)$ & C34-C33-H33a & $108.9(2)$ \\
\hline C9-C10-C11 & $121.19(20)$ & C34-C33-H33b & $107.4(3)$ \\
\hline O2-C10-C11 & $119.3(2)$ & H33a-C33-H33b & $109.5(3)$ \\
\hline C10-C11-C12 & $124.2(2)$ & C33-C34-C35 & $114.6(2)$ \\
\hline
\end{tabular}




$\begin{array}{llll}\text { C10-C11-C16 } & 117.8(2) & \text { C33-C34-H34a } & 107.5(2) \\ \text { C12-C11-C16 } & 117.9(2) & \text { C33-C34-H34b } & 108.8(3) \\ \text { C11-C12-C13 } & 121.2(2) & \text { C35-C34-H34a } & 107.6(3) \\ \text { C11-C12-H12 } & 119.3(2) & \text { C35-C34-H34b } & 108.8(2) \\ \text { C13-C12-H12 } & 119.5(2) & \text { H34a-C34-H34b } & 109.5(3) \\ \text { C12-C13-C14 } & 119.9(2) & \text { C34-C35-C36 } & 114.0(2) \\ \text { C12-C13-H13 } & 120.3(2) & \text { C34-C35-H35a } & 107.7(2) \\ \text { C14-C13-H13 } & 119.8(2) & \text { C34-C35-H35b } & 108.9(2) \\ \text { C13-C14-C15 } & 119.6(2) & \text { C36-C35-H35a } & 108.0(2) \\ \text { C13-C14-O3 } & 124.5(2) & \text { C36-C35-H35b } & 108.7(2) \\ \text { C15-C14-O3 } & 115.9(2) & \text { H35a-C35-H35b } & 109.5(2) \\ \text { C14-C15-C16 } & 120.2(2) & \text { B1-C36-C35 } & 106.9(2) \\ \text { C14-C15-H15 } & 119.7(3) & \text { B1-C36-C37 } & 112.45(19) \\ \text { C16-C15-H15 } & 120.1(3) & \text { B1-C36-H36 } & 107.4(2) \\ \text { C11-C16-C15 } & 121.2(2) & \text { C35-C36-C37 } & 111.83(19) \\ \text { C11-C16-H16 } & 119.2(2) & \text { C35-C36-H36 } & 109.2(2) \\ \text { C15-C16-H16 } & 119.7(2) & \text { C37-C36-H36 } & 109.0(2) \\ \text { C14-O3-C17 } & 117.3(2) & \text { C36-C37-C38 } & 116.1(2) \\ \text { O3-C17-H17a } & 109.5(4) & \text { C36-C37-H37a } & 108.0(2) \\ \text { O3-C17-H17b } & 109.5(4) & \text { C36-C37-H37b } & 107.9(2) \\ \text { O3-C17-H17c } & 109.5(3) & \text { C38-C37-H37a } & 107.9(2) \\ \text { H17a-C17-H17b } & 109.5(11) & \text { C38-C37-H37b } & 107.4(2) \\ \text { H17a-C17-H17c } & 109.5(12) & \text { H37a-C37-H37b } & 109.5(2) \\ \text { H17b-C17-H17c } & 109.5(13) & \text { C37-C38-C39 } & 115.65(19) \\ \text { C5-C18-C19 } & 123.0(2) & \text { C37-C38-H38a } & 107.5(2) \\ \text { C5-C18-C23 } & 118.2(2) & \text { C37-C38-H38b } & 108.3(2) \\ \text { C19-C18-C23 } & 118.6(2) & \text { C39-C38-H38a } & 107.6(2) \\ \text { C18-C19-C20 } & 120.2(2) & \text { C39-C38-H38b } & 108.2(2) \\ \text { C18-C19-H19 } & 119.6(2) & \text { H38a-C38-H38b } & 109.5(2) \\ \text { C20-C19-H19 } & 120.1(3) & \text { C32-C39-C38 } & 115.68(20) \\ \text { C19-C20-C21 } & 120.2(2) & \text { C32-C39-H39a } & 108.1(2) \\ \text { C19-C20-H20 } & 120.0(3) & \text { C32-C39-H39b } & 107.8(2) \\ \text { C21-C20-H20 } & 119.9(3) & \text { C38-C39-H39a } & 108.0(2) \\ \text { C20-C21-C22 } & 120.0(3) & \text { C38-C39-H39b } & 107.7(2) \\ \text { C20-C21-H21 } & 119.8(3) & \text { H39a-C39-H39b } & 109.5(2) \\ & & & \end{array}$




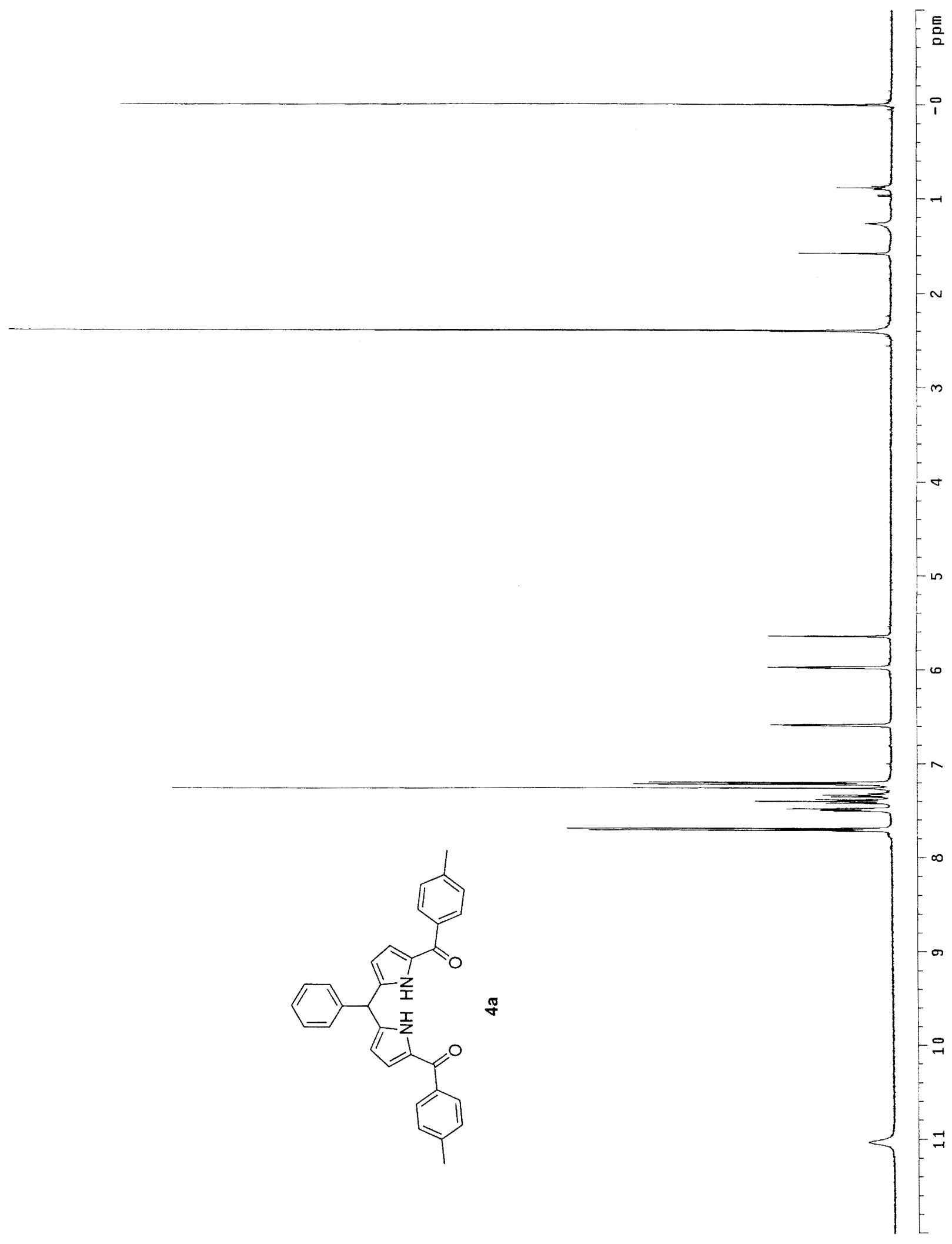

S-18 


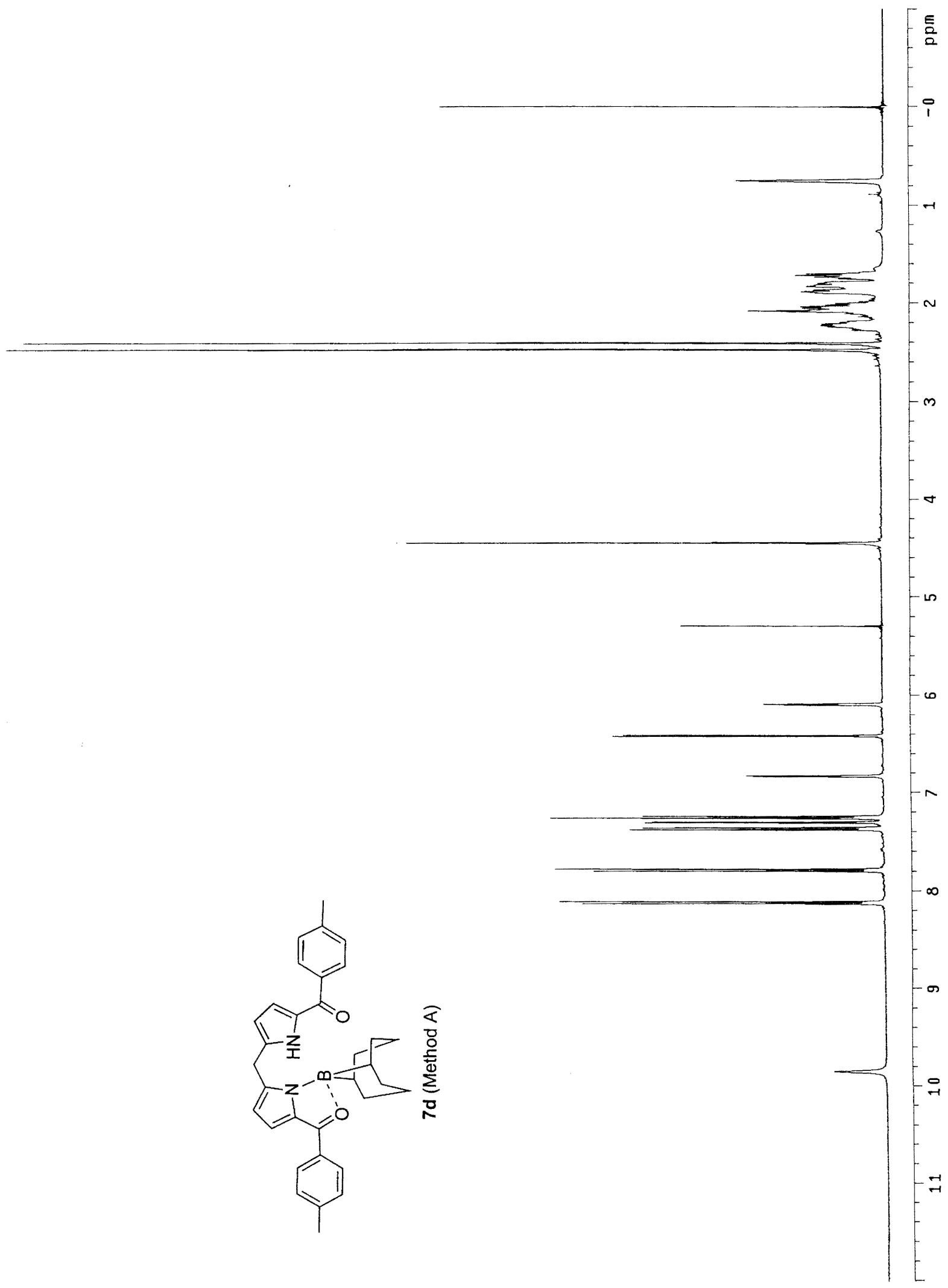

S-19 


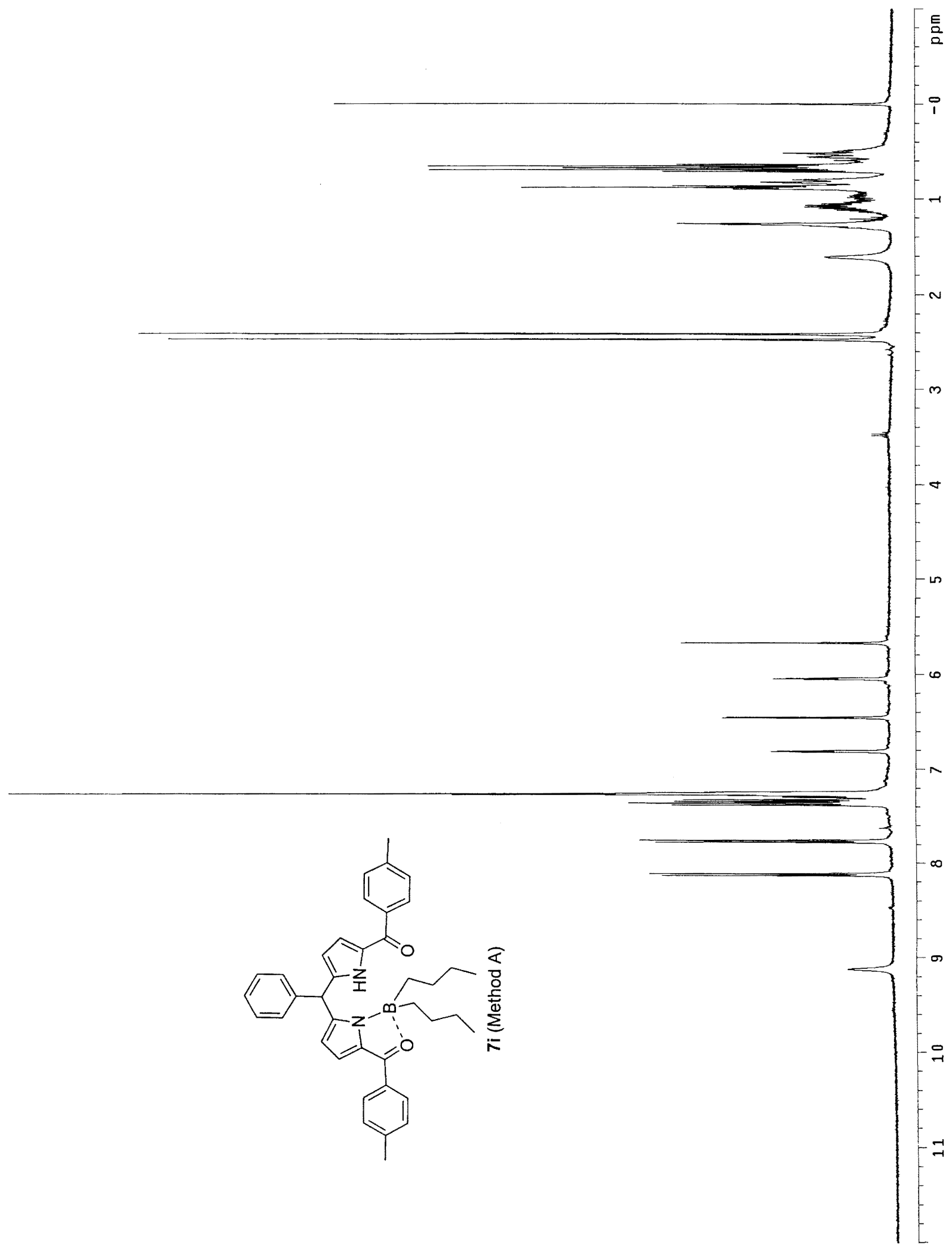




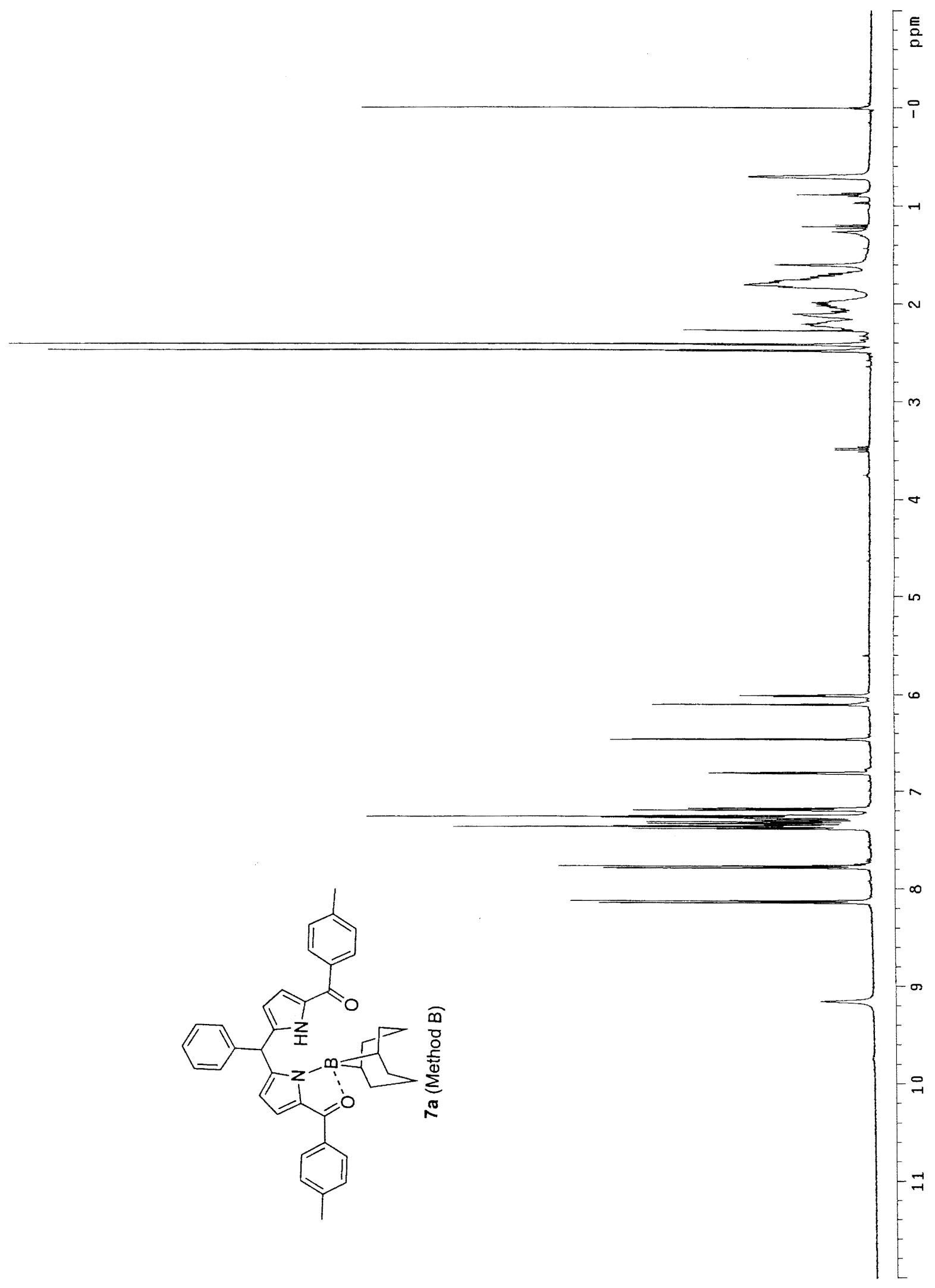




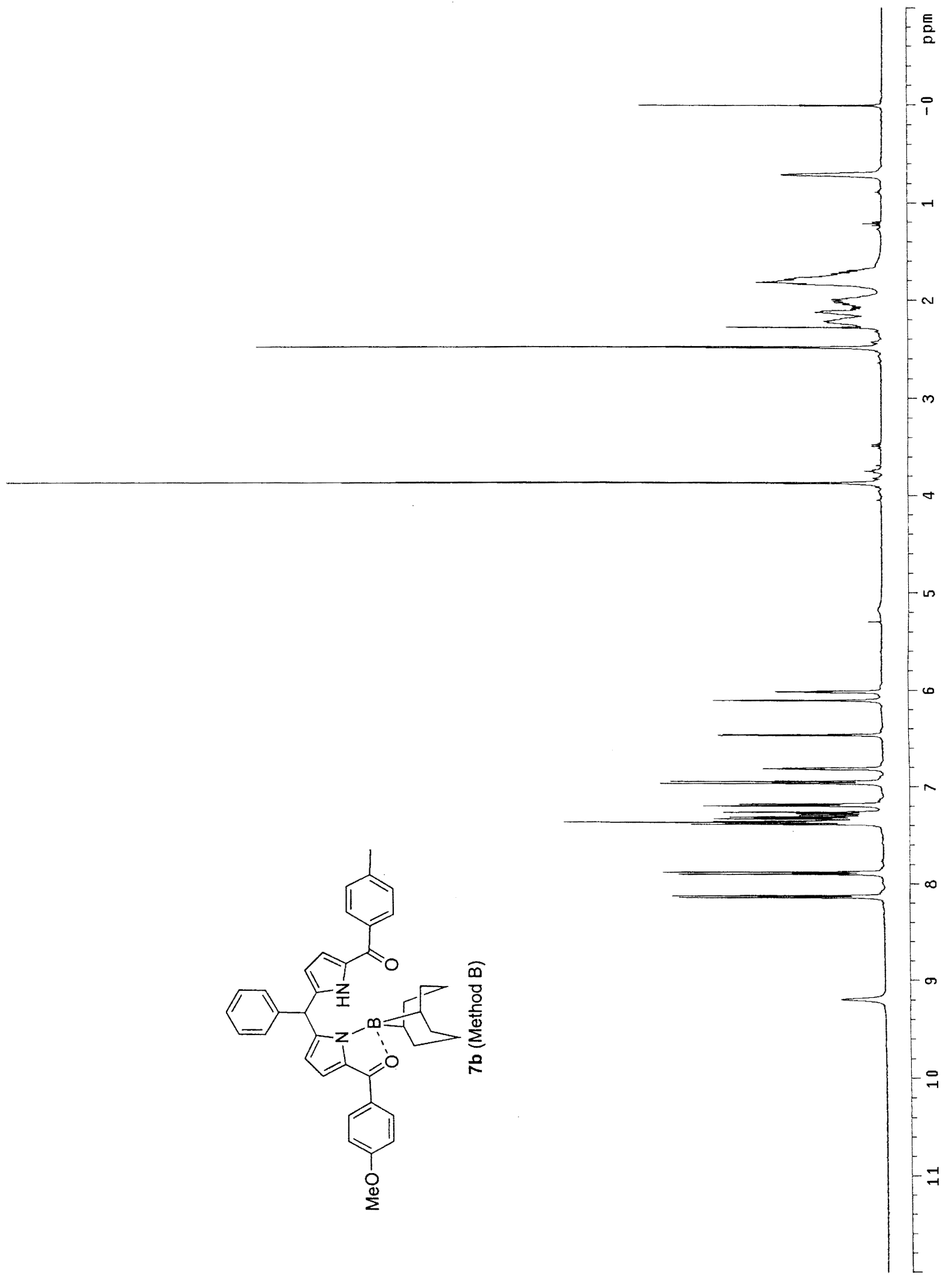




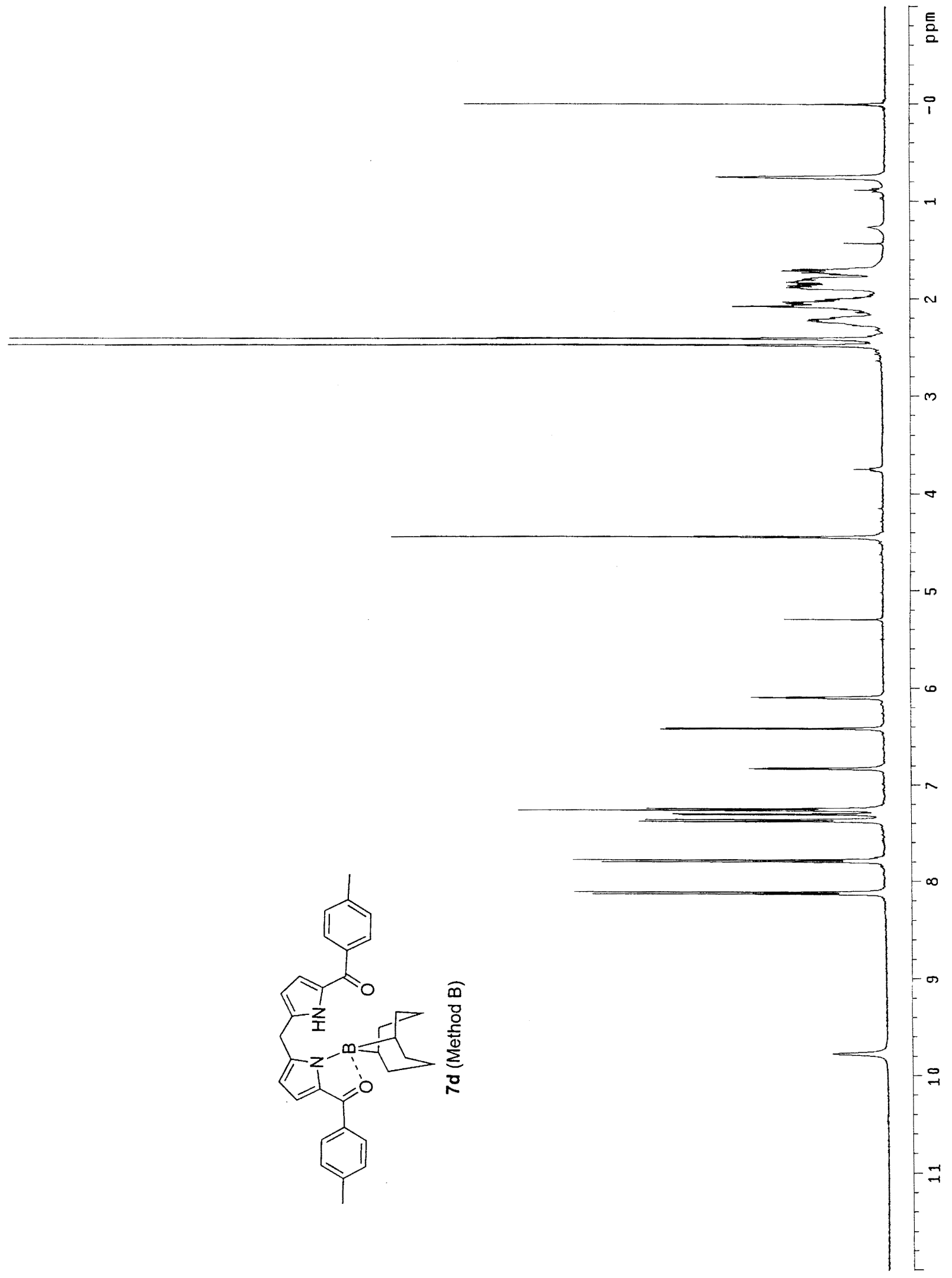




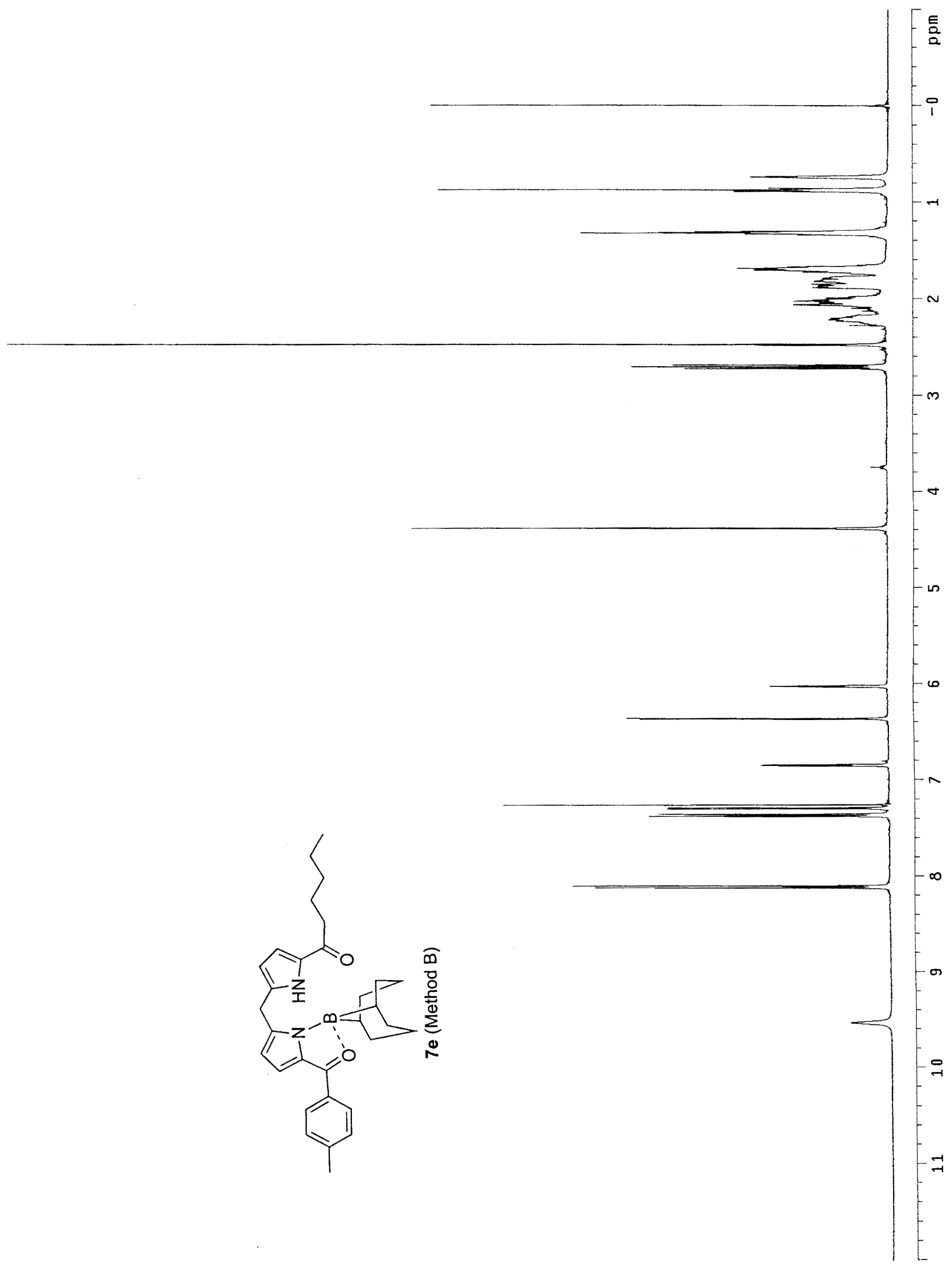




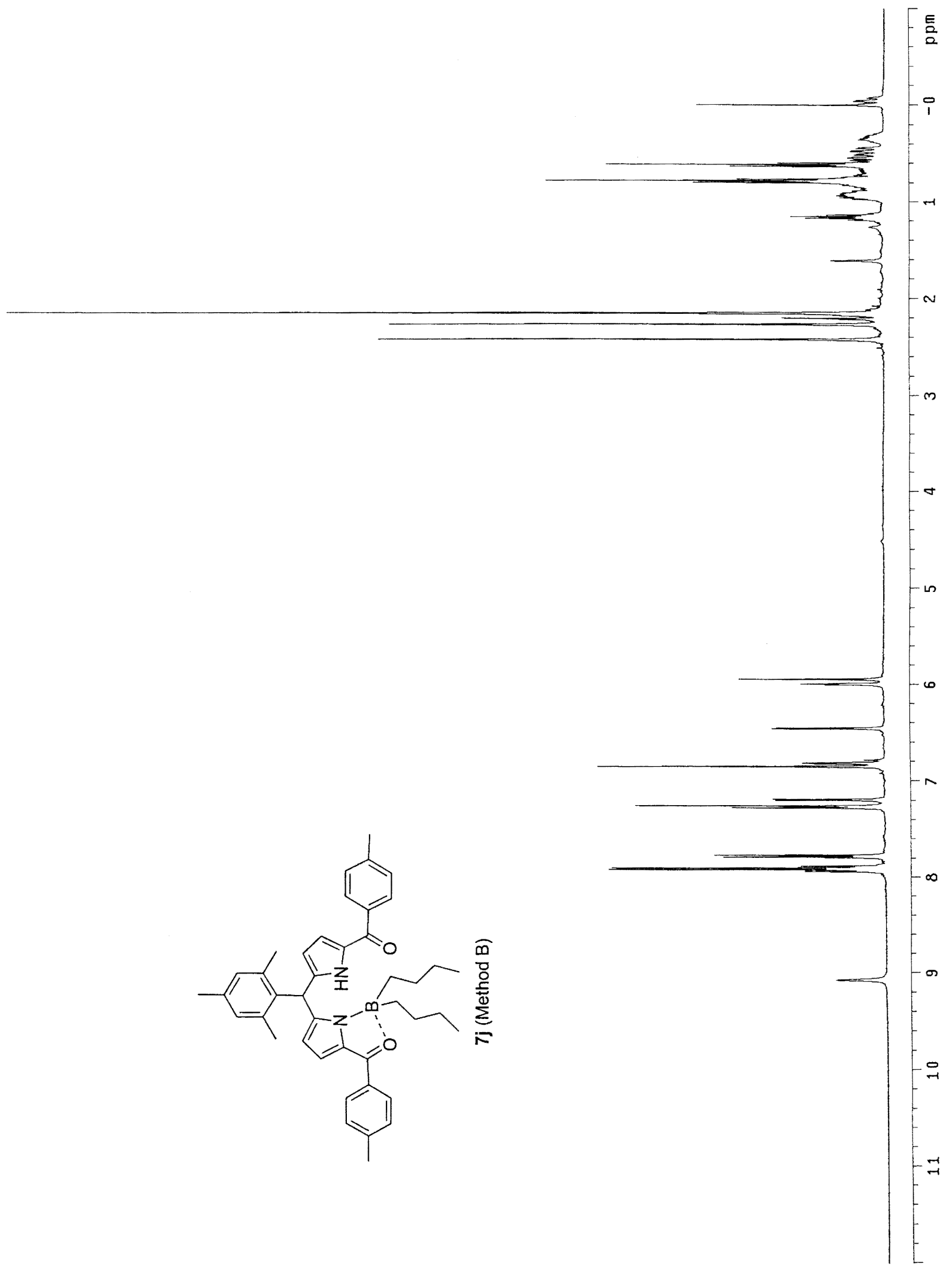




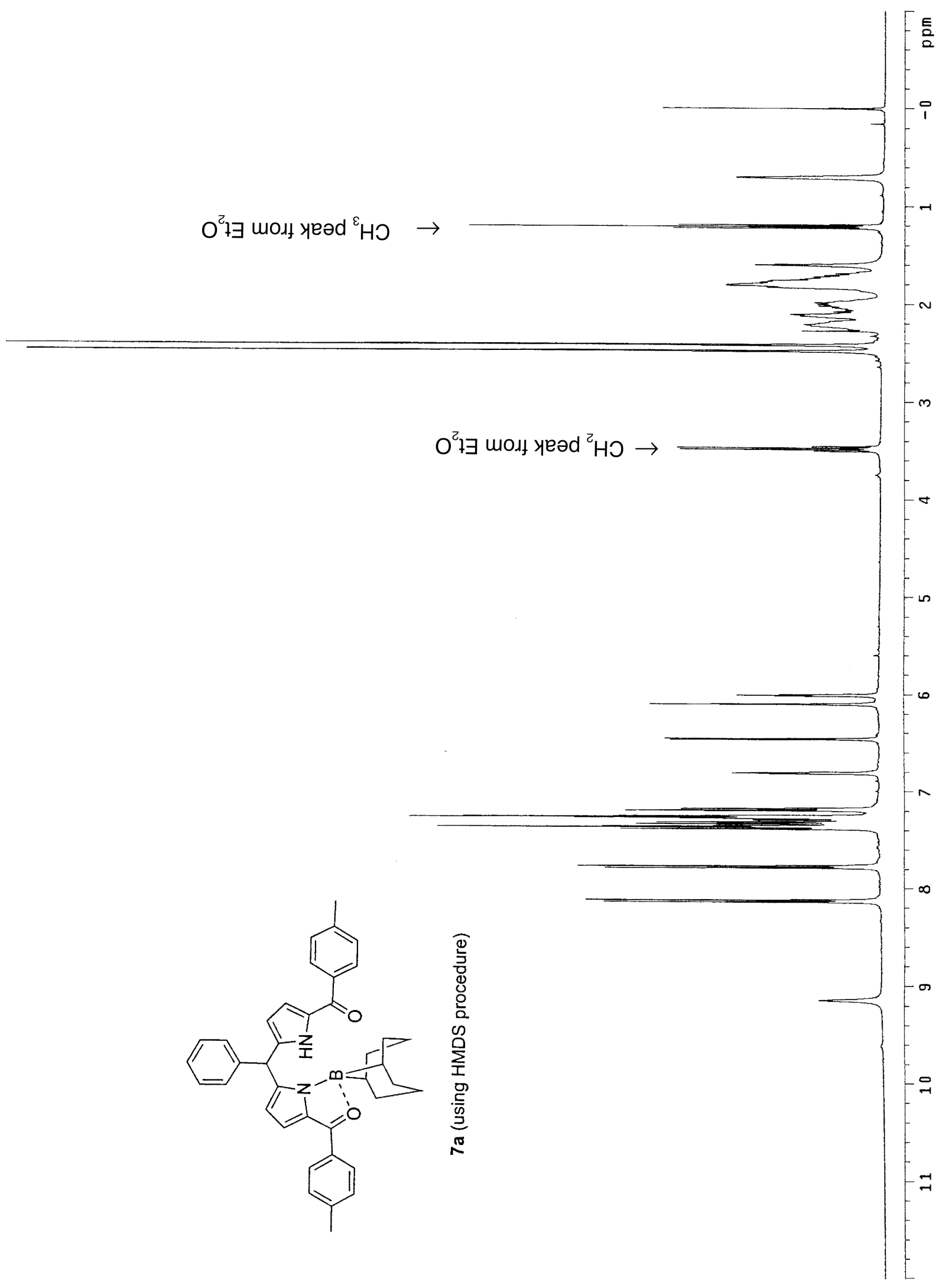




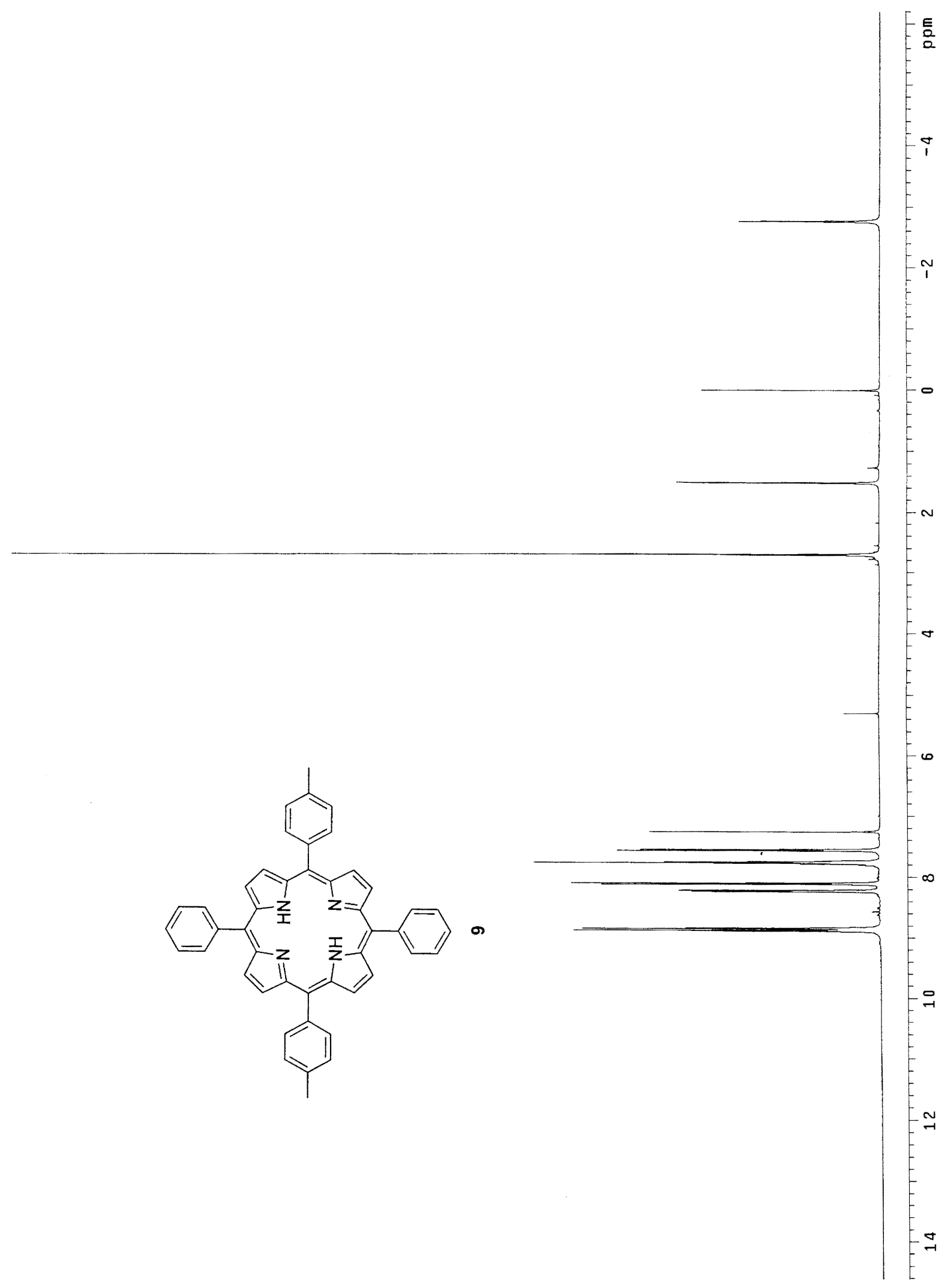




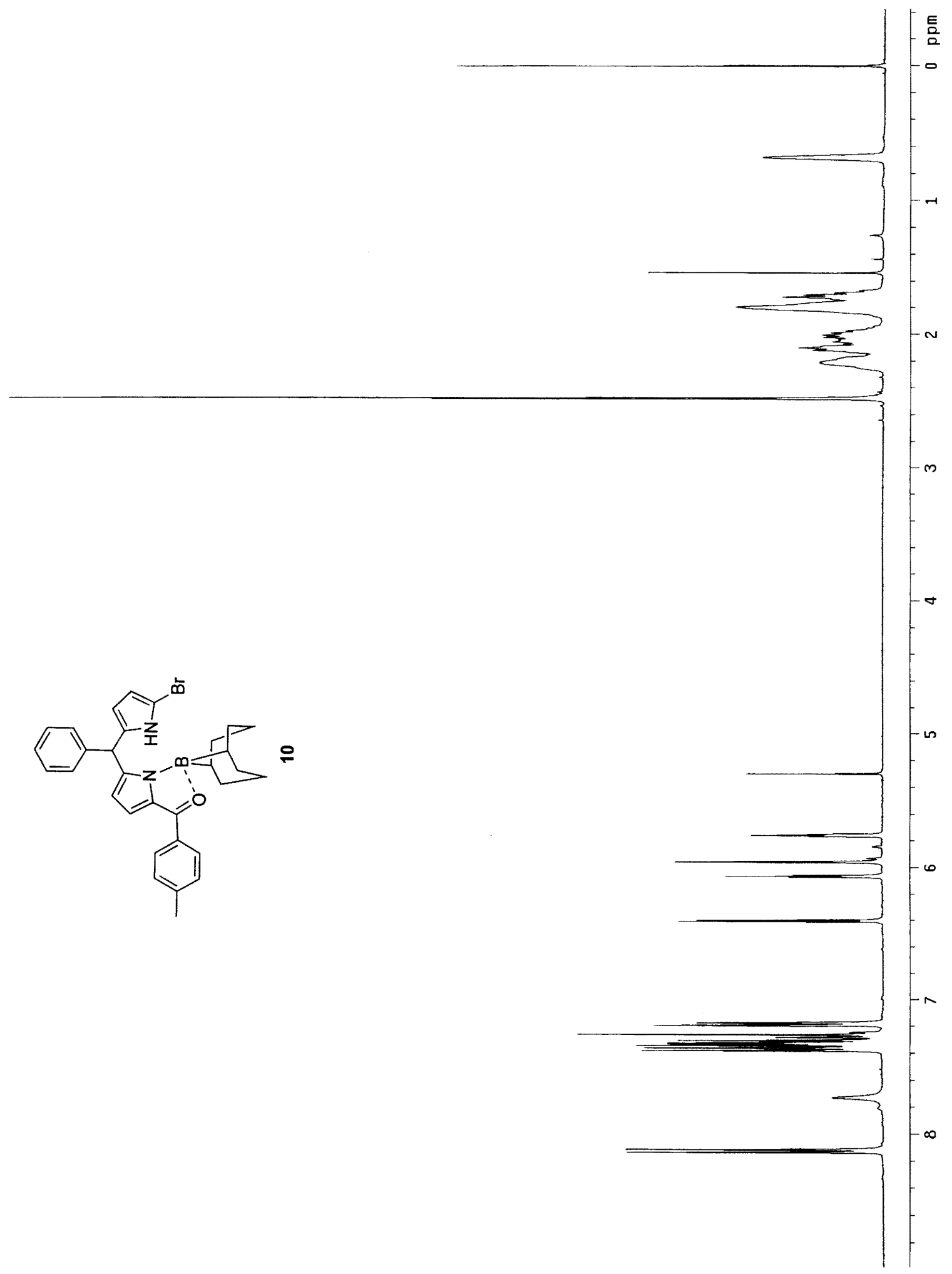

